\title{
Formation of Bicyclic Ethers from Lewis Acid Promoted Cyclizations of Cyclic Oxonium Ions
}

\author{
Pradip K. Sasmal and Martin E. Maier*
}

Institut für Organische Chemie, Universität Tübingen, Auf der Morgenstelle 18, D-72076 Tübingen, Germany

martin.e.maier@uni-tuebingen.de

Supporting Information 


\section{Experimental Section}

General. ${ }^{1} \mathrm{H}$ and ${ }^{13} \mathrm{C}$ NMR: Bruker AC 250, Bruker Avance 400, spectra were recorded in $\mathrm{CDCl}_{3}$; chemical shifts are calibrated to the residual proton and carbon resonance in $\mathrm{CDCl}_{3}(7.25 \mathrm{ppm}, 77.00$ ppm). EI-MS; Finnigan Triple-Stage-Quadrupol (TSQ-70). HRMS (EI): modified AMD Intectra MAT 711 A. Flash chromatography: J. T. Baker silica gel 43-60 $\mu \mathrm{m}$. Thin-layer chromatography MacheryNagel Polygram Sil G/UV ${ }_{254}$. Solvents were distilled prior to use; petroleum ether with a boiling range of $40-60{ }^{\circ} \mathrm{C}$ was used.

General procedure for Grignard reaction. Under nitrogen atmosphere, a stirred solution of aldehyde 1a-c (1 eq) in THF was treated dropwise with a solution of vinylmagnesium bromide in THF (1.1 eq) at $0{ }^{\circ} \mathrm{C}$. The cooling bath was removed and the reaction mixture stirred at room temperature for $6 \mathrm{~h}$. The reaction mixture was quenched with aqueous $\mathrm{NH}_{4} \mathrm{Cl}$ solution and extraced with ether three times. The combined organic layers were washed with water, brine, and dried $\left(\mathrm{Na}_{2} \mathrm{SO}_{4}\right)$, filtered, and concentrated with a rotavapor to give colorless viscous oils $\mathbf{2 a - d}$. The crude product was pure enough for the next step.

General procedure for cyclic acetal formation. To a solution of the allyl alcohol derivatives 2a-d in dichloromethane $(0.06 \mathrm{M})$ was added camphor-10-sulphonic acid (10 mol \%) at room temperature. After stirring the reaction mixture at the same temperature for $5 \mathrm{~h}$, solid $\mathrm{NaHCO}_{3}(5 \mathrm{eq})$ was added, and stirring was continued for $15 \mathrm{~min}$. Then the mixture was filtered and washed with dichloromethane. The combined filtrate and washings were concentrated and the crude product was sujected to flash chromatography using pentane/ether as eluent to give the cycli hemiacetals 3a-d as thin oils (82-91\% yield for two steps).

General procedure for Suzuki reaction. To a stirred solution of the olefin (3a-d, 10) (1 eq) in THF was added $9 \mathrm{BBN}(0.5 \mathrm{M}$ in THF, $1.2 \mathrm{eq})$ at $0{ }^{\circ} \mathrm{C}$. The reaction mixture was stirred at the same temperature for $1 \mathrm{~h}$ and then at room temperature for $3 \mathrm{~h}$. The excess borane reagent was quenched with degassed water ( $1 \mathrm{eq}$ ) and diluted with benzene (double of the total volume of THF). To the mixture were added aqueous $3 \mathrm{~N} \mathrm{NaOH}(4 \mathrm{eq}), \mathrm{Pd}\left(\mathrm{PPh}_{3}\right)_{3}(3 \mathrm{~mol} \%)$, and the vinyl bromide (6a or $\left.\mathbf{6 b}\right)(1 \mathrm{eq})$ 
and the reaction mixture was refluxed for $4 \mathrm{~h}$. After cooling to room temperature the organic layer was separated and the aqueous layer was extracted with ether twice. The combined organic layers were washed with water, brine, dried $\left(\mathrm{Na}_{2} \mathrm{SO}_{4}\right)$, filtered, and concentrated in vacuo. Purification by flash chromatography (ethyl acetate/hexane) afforded the alkenes 7a-d, 8a-d, 9a-d as pale yellow viscous oils $(59-79 \%$ yield).

General procedure for bicyclic ether formation. To a stirred solution of the acetal-alkene derivative (7a-d, 8a-d, 8aOEt, 9a-d, 11, 12) $\left(0.02 \mathrm{M}\right.$ in the reported solvent) was added $\mathrm{SnCl}_{4}(1.5 \mathrm{eq}$, 1.0 $\mathrm{M}$ in dichlomethane) or TMSOTf $(1.5 \mathrm{eq})$ or $\mathrm{BF}_{3} \cdot \mathrm{Et}_{2} \mathrm{O}(2.0 \mathrm{eq})$ at $-78{ }^{\circ} \mathrm{C}$. After stirring for $1 \mathrm{~h}$ at the same temperature, the reaction mixture was slowly warmed up to $-10{ }^{\circ} \mathrm{C}$ over a period of $4 \mathrm{~h}$ and then quenched with $2 \mathrm{~N}$ aq $\mathrm{NaOH}$ solution. The organic layer was separated and the aqueous layer was extracted with dichloromethane twice. The combined organic layers were washed with water, brine, dried $\left(\mathrm{Na}_{2} \mathrm{SO}_{4}\right)$, filtered, and concentrated in vacuo using a rotavapor. The crude products were subjected to flash chromatography (ethyl acetate/hexane) to furnish the bicyclic ethers.

Hydrolysis of the thio enol ether 22: To a stirred solution of the alkenyl phenyl sulfide 22 in THF was added aqueous $\mathrm{HCl}$ solution $(3 \mathrm{~N}, 3 \mathrm{eq})$ at $0{ }^{\circ} \mathrm{C}$ and the reaction mixture was stirred overnight at room temperature. Solid $\mathrm{NaHCO}_{3}$ was added to neutralize the acid. The reaction mixture was treated with water and was extracted with ether three times. The combined organic layers were washed with water, brine, dried over $\mathrm{Na}_{2} \mathrm{SO}_{4}$, filtered and concentrated in vacuo. Chromatographic purification furnished the desired ketone $\mathbf{2 3}$ as a colorless oil (90\% yield).

$\left(1 S^{*}, 2 S^{*}\right)$-2-methyl-4-oxo-5-cyclodecen-1-yl acetate (24). To a solution of LDA in THF (2 mL) [generated from ${ }^{\mathrm{n}} \mathrm{BuLi}(2.5 \mathrm{eq})$ and ${ }^{\mathrm{i}} \mathrm{Pr}_{2} \mathrm{NH}(3.0 \mathrm{eq})$ at $-10{ }^{\circ} \mathrm{C}$ ] at $0{ }^{\circ} \mathrm{C}$ was added a solution of $\left(1 \mathrm{R}^{*}, 5 \mathrm{~S}^{*}, 6 \mathrm{~S}^{*}\right)-5$-methyl-11-oxabicyclo[4.4.1]undecan-3-one (23) (40 mg, $\left.0.22 \mathrm{mmol}\right)$ in THF (1 mL). The reaction mixture was warmed to room temperature and stirred for $48 \mathrm{~h}$ and then quenched with aqueous $\mathrm{NH}_{4} \mathrm{Cl}$ solution and extraced with ether thrice. The combined organic layer was washed with water, brine, dried $\left(\mathrm{Na}_{2} \mathrm{SO}_{4}\right)$, filtered, and concentrated in vacuo. The residue was dissolved in dichloromethane and treated with DMAP (1 eq), triethylamine (10 eq) and acetic anhydride (6 eq) at 0 
${ }^{\circ} \mathrm{C}$. The reaction mixture was warmed to room temperature and stirred for $2 \mathrm{~h}$. Then it was quenched with aqueous $\mathrm{NaHCO}_{3}$ solution and extracted with dichloromethane. The dichloromethane layer was washed with water, brine, dried over $\mathrm{Na}_{2} \mathrm{SO}_{4}$, and filtered. Concentration and chromatographic purification afforded the desired acetate $\mathbf{2 4}$ as a colorless viscous oil (74\% yield) together with the starting ketone $\mathbf{2 3}$ (22\%).

\section{Spectral Data}

Compound 2d: ${ }^{1} \mathrm{H}$ NMR (400 MHz): $\delta 4.90(\mathrm{~s}, 1 \mathrm{H}), 4.80(\mathrm{~s}, 1 \mathrm{H}), 4.33(\mathrm{t}, J=5.8 \mathrm{~Hz}, 1 \mathrm{H}), 4.02(\mathrm{t}, J=$ $6.5 \mathrm{~Hz}, 1 \mathrm{H}), 3.28(\mathrm{~s}, 6 \mathrm{H}), 1.69(\mathrm{~s}, 3 \mathrm{H}), 1.62-1.48(\mathrm{~m}, 4 \mathrm{H}), 1.43-1.22(\mathrm{~m}, 4 \mathrm{H}) ;{ }^{13} \mathrm{C} \mathrm{NMR}(100 \mathrm{MHz}): \delta$ $147.6,110.9,104.4,75.8,52.6,52.5,34.8,32.4,25.4,24.5,17.4$; IR (neat): 3444, 2844, 1651, 1454, $1386,1129,1052 \mathrm{~cm}^{-1}$.

Compound 3d: ${ }^{1} \mathrm{H}$ NMR (400 MHz): $\delta 4.95(\mathrm{~s}, 1 \mathrm{H}), 4.75(\mathrm{~s}, 1 \mathrm{H}), 4.65(\mathrm{dd}, J=5.5,8.5 \mathrm{~Hz}, 1 \mathrm{H}-$ major), 4.49 (dd, $J=3.5,7.5 \mathrm{~Hz}, 1 \mathrm{H}$-minor), 4.15 (d, br., $J=10.5 \mathrm{~Hz}, 1 \mathrm{H}$-major), 3.89 (dd, $J=4.0,9.0$ Hz, 1H-minor), 3.39 (s, 3H-minor), 3.30 (s, 3H-major), 2.14-2.05 (m, 1H), 1.96-1.84 (m, 1H), 1.75 (s, 3H-minor), 1.74 (s, 3H-major), 1.82-1.24 (m, 6H); ${ }^{13} \mathrm{C}$ NMR (100 MHz), major isomer: $\delta$ 146.8, 109.9, $102.4,73.7,55.1,35.2,34.7,29.7,22.5,18.9$; minor isomer: $\delta 146.3,109.9,106.1,80.2,55.6,35.9$, 35.0, 25.2, 22.9, 19.1; IR (neat): 2934, 1652, 1452, 1371, 1204, 1132, $1066 \mathrm{~cm}^{-1}$.

Compound 9d: ${ }^{1} \mathrm{H}$ NMR (400 MHz), major isomer: $\delta$ 7.47-7.40 (m, 2H), 7.35-7.24 (m, 3H), 5.11 (s, $1 \mathrm{H}), 4.85(\mathrm{~s}, 1 \mathrm{H}), 4.59(\mathrm{dd}, J=5.5,9.0 \mathrm{~Hz}, 1 \mathrm{H}), 3.68(\mathrm{dd}, J=3.5,10.0 \mathrm{~Hz}, 1 \mathrm{H}), 3.36(\mathrm{~s}, 3 \mathrm{H}), 2.42(\mathrm{dd}$, $J=3.0,12.0 \mathrm{~Hz}, 1 \mathrm{H}), 2.07-1.93(\mathrm{~m}, 3 \mathrm{H}), 1.88-1.77(\mathrm{~m}, 1 \mathrm{H}), 1.65-1.50(\mathrm{~m}, 3 \mathrm{H}), 1.47-1.16(\mathrm{~m}, 3 \mathrm{H})$, $0.92(\mathrm{~d}, J=6.5 \mathrm{~Hz}, 3 \mathrm{H}) ;{ }^{13} \mathrm{C} \mathrm{NMR}(100 \mathrm{MHz}): \delta 145.1,133.5,133.0,129.0,127.8,113.3,102.9,73.8$, 55.5, 39.8, 37.5, 34.9, 31.5, 29.3, 22.6, 15.1; IR (neat): 2932, 1607, 1439, 1066, $1025 \mathrm{~cm}^{-1}$; EIMS, $m / z$ : $306[\mathrm{M}]^{+}, 275[\mathrm{M}-\mathrm{OMe}]^{+}, 197,177,156,149 ;$ HRMS: calcd for $\mathrm{C}_{18} \mathrm{H}_{26} \mathrm{O}_{2} \mathrm{~S}$ 306.1654, found 306.1668 .

Compound 22a: ${ }^{1} \mathrm{H}$ NMR (400 MHz), major isomer: $\delta 7.40-7.15(\mathrm{~m}, 5 \mathrm{H}), 5.85(\mathrm{dd}, J=2.8,4.5 \mathrm{~Hz}$, $1 \mathrm{H}), 4.49-4.38(\mathrm{~m}, 1 \mathrm{H}), 3.62(\mathrm{ddd}, J=5.0,5.0,9.5 \mathrm{~Hz}, 1 \mathrm{H}), 2.72(\mathrm{dddd}, J=2.3,2.3,11.0,16.0 \mathrm{~Hz}$, 
1H), 2.30-2.17 (m, 1H), 2.16-1.98 (m, 1H), $2.04(\mathrm{dd}, J=1.8,16.0 \mathrm{~Hz}, 1 \mathrm{H}), 1.90-1.45(\mathrm{~m}, 7 \mathrm{H}), 0.81(\mathrm{~d}$, $J=6.8,3 \mathrm{H}) ;{ }^{13} \mathrm{C}$ NMR $(100 \mathrm{MHz}): \delta 136.8,135.1,132.6,130.6,129.0,126.7,84.0,76.5,40.9,37.9$, 37.3, 33.9, 25.6, 25.5, 20.2; IR (neat): 2925, 1582, 1477, 1438, 1157, 1129, $1024 \mathrm{~cm}^{-1}$; EIMS, m/z: 274 (M), 232, 197, 189, 165; HRMS: calcd for $\mathrm{C}_{17} \mathrm{H}_{22} \mathrm{OS} 274.1391$, found 274.1406.

Compound 23: ${ }^{1} \mathrm{H}$ NMR (400 MHz): $\delta 4.15$ (dddd, $\left.J=5.0,6.5,10.0,15.0 \mathrm{~Hz}, 1 \mathrm{H}\right), 3.73$ (ddd, $J=$ 5.0, 5.0, $9.5 \mathrm{~Hz}, 1 \mathrm{H}), 2.74$ (ddd, $J=1.0,7.0,13.0 \mathrm{~Hz}, 1 \mathrm{H}), 2.62(\mathrm{dd}, J=10.5,13.0 \mathrm{~Hz}, 1 \mathrm{H}), 2.55$ (ddd, $J=1.5,5.0,13.0 \mathrm{~Hz}, 1 \mathrm{H}), 2.34(\mathrm{ddd}, J=2.0,2.0,13.0 \mathrm{~Hz}, 1 \mathrm{H}), 2.06(\mathrm{~m}, 1 \mathrm{H}), 1.94-1.66(\mathrm{~m}, 5 \mathrm{H}), 1.64-$ $1.44(\mathrm{~m}, 3 \mathrm{H}), 0.94(\mathrm{~d}, J=6.5 \mathrm{~Hz}, 3 \mathrm{H}) ;{ }^{13} \mathrm{C} \mathrm{NMR}(100 \mathrm{MHz}): \delta 210.0,82.9,72.5,50.5,49.7,36.2$, 33.4, 32.5, 25.5, 24.8, 20.3; IR (neat): 2927, 1704, 1462, 1141, $972 \mathrm{~cm}^{-1}$; EIMS, m/z: 182 (M), 165, 154, 139, 123, 111, 97; HRMS: calcd for $\mathrm{C}_{11} \mathrm{H}_{18} \mathrm{O}_{2}$ 182.1307, found 182.1314 .

Compound 24: ${ }^{1} \mathrm{H}$ NMR (400 MHz): $\delta 6.32(\mathrm{~d}, J=12.0 \mathrm{~Hz}, 1 \mathrm{H}), 5.76(\mathrm{ddd}, J=5.5,12.0,12.0 \mathrm{~Hz}$, 1H), $4.70(\mathrm{ddd}, J=1.8,3.5,12.0 \mathrm{~Hz}, 1 \mathrm{H}), 2.81-2.69(\mathrm{~m}, 1 \mathrm{H}), 2.69(\mathrm{~d}, J=14.0 \mathrm{~Hz}, 1 \mathrm{H}), 2.68-2.58(\mathrm{~m}$, 1H), $2.16(\mathrm{dd}, J=1.0,14.0 \mathrm{~Hz}, 1 \mathrm{H}), 2.10(\mathrm{~m}, 1 \mathrm{H}), 2.04(\mathrm{~s}, 3 \mathrm{H}), 1.60-1.23(\mathrm{~m}, 5 \mathrm{H}), 1.14-1.02(\mathrm{~m}, 1 \mathrm{H})$, $1.00(\mathrm{~d}, J=6.8 \mathrm{~Hz}, 3 \mathrm{H}) ;{ }^{13} \mathrm{C} \mathrm{NMR}(100 \mathrm{MHz}): \delta 206.7,170.7,138.6,132.9,76.8,46.6,31.7,27.2$, 24.4, 23.6, 21.1, 20.1, 20.0; IR (neat): 2933, 1736, 1687, 1625, 1469, 1369, $1245 \mathrm{~cm}^{-1}$; EIMS, m/z: $224[\mathrm{M}]^{+}, 182,164,135,121,107,84$; HRMS: calcd for $\mathrm{C}_{13} \mathrm{H}_{20} \mathrm{O}_{3} 224.1412$, found 224.1425. 

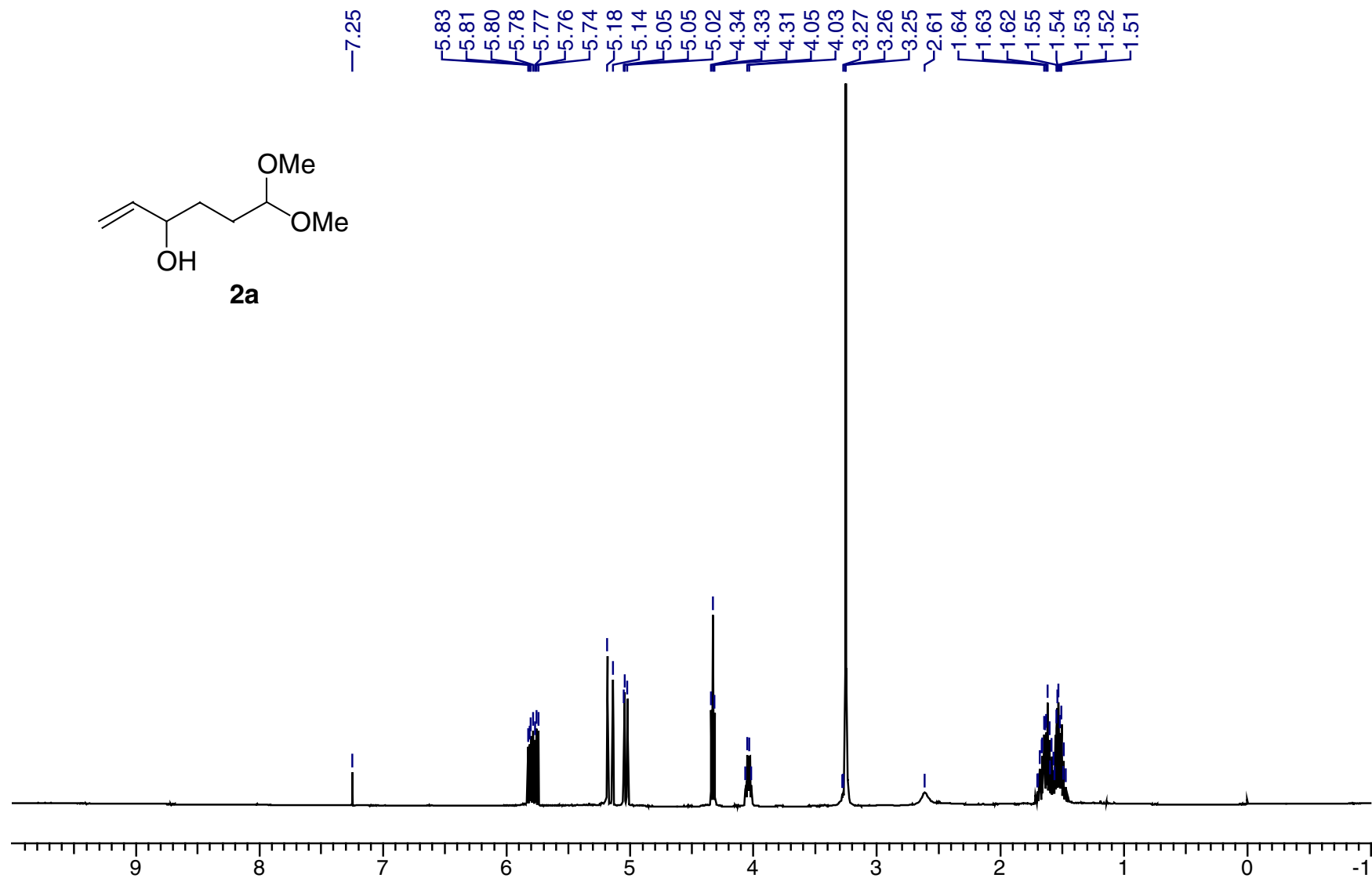

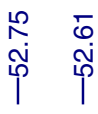

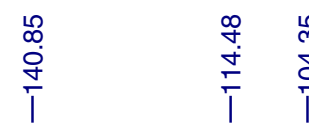

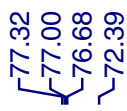

$\stackrel{\sim}{\wedge}$

กิ่ กิ่

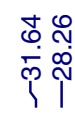
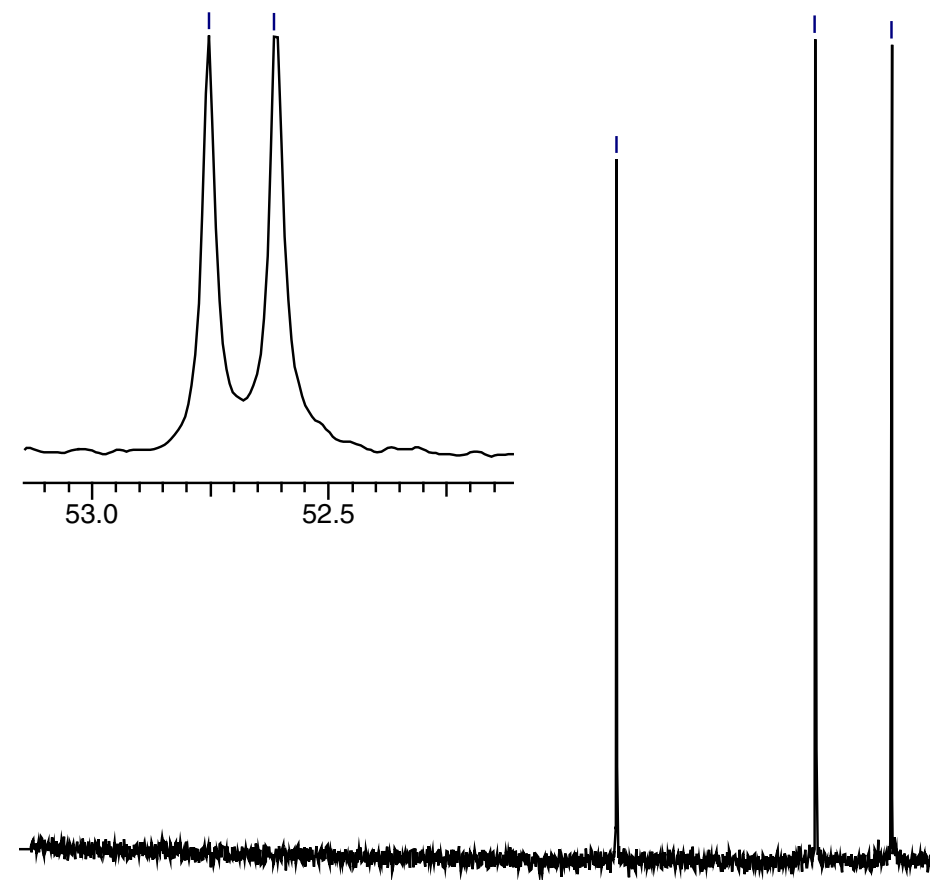

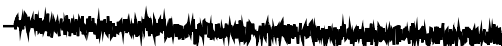

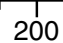

150

50 


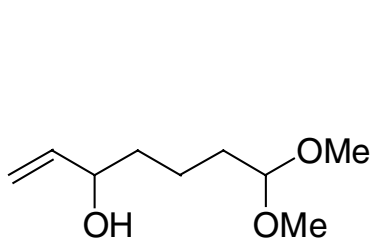

2b

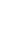
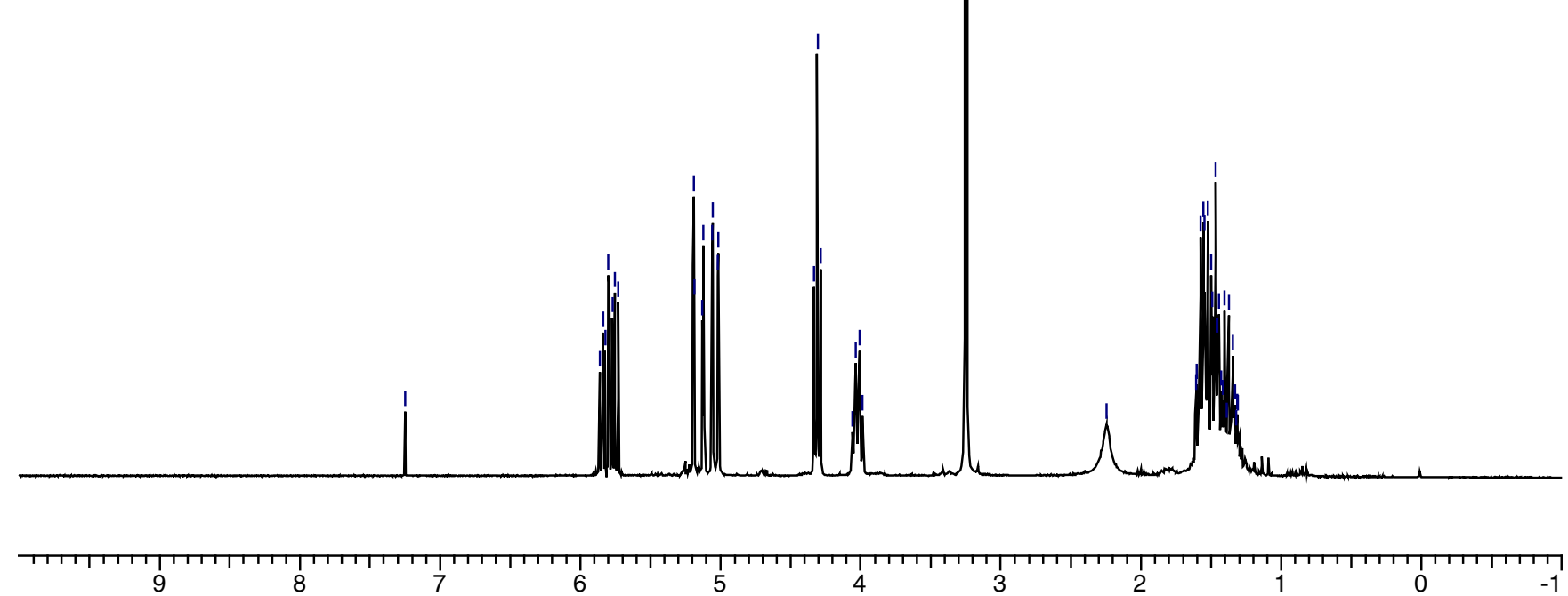

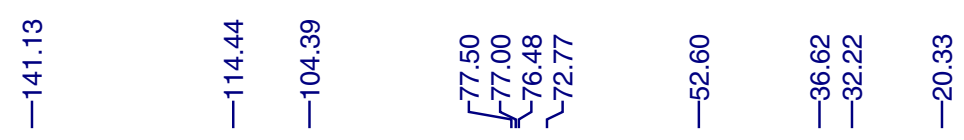
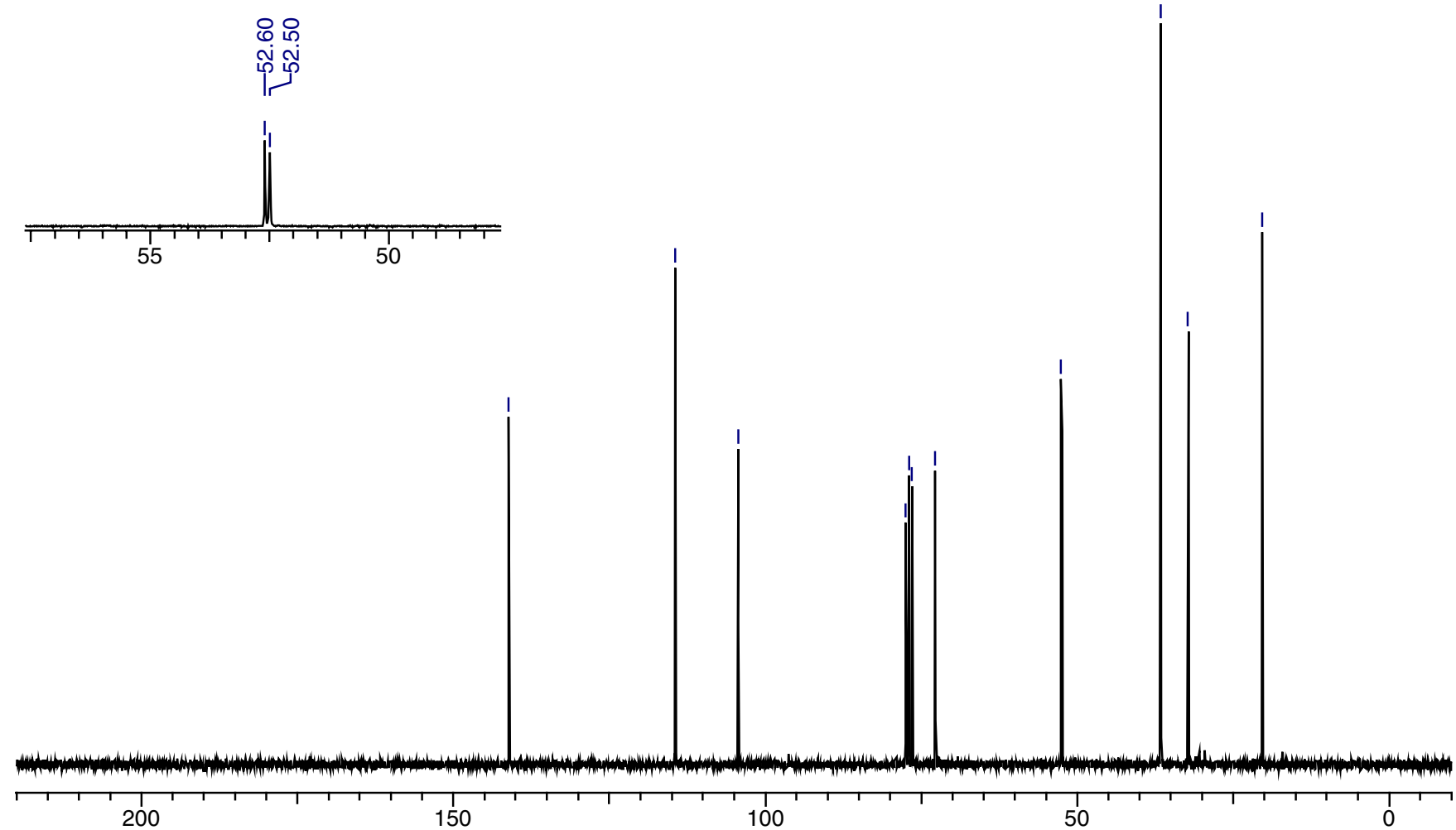

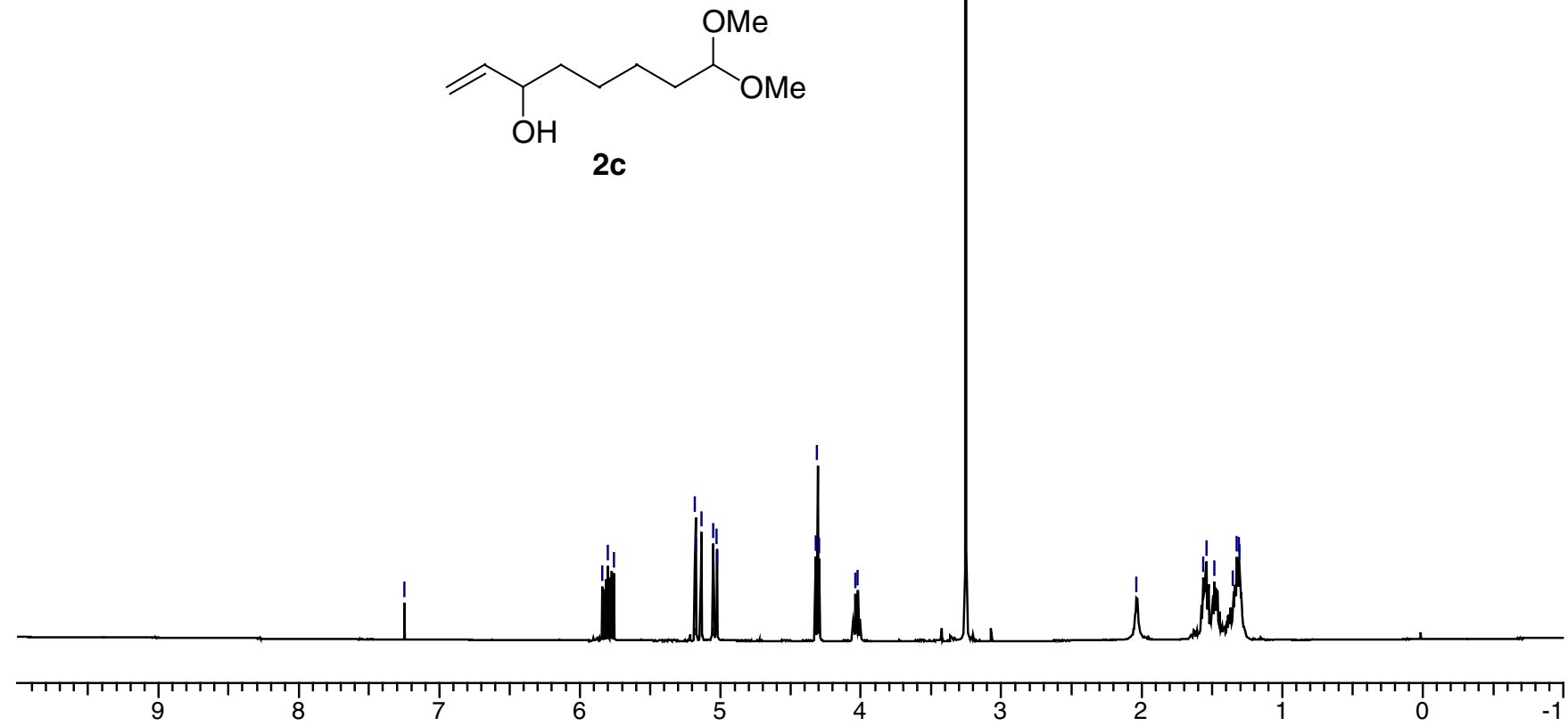

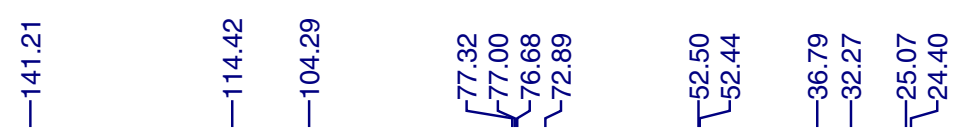

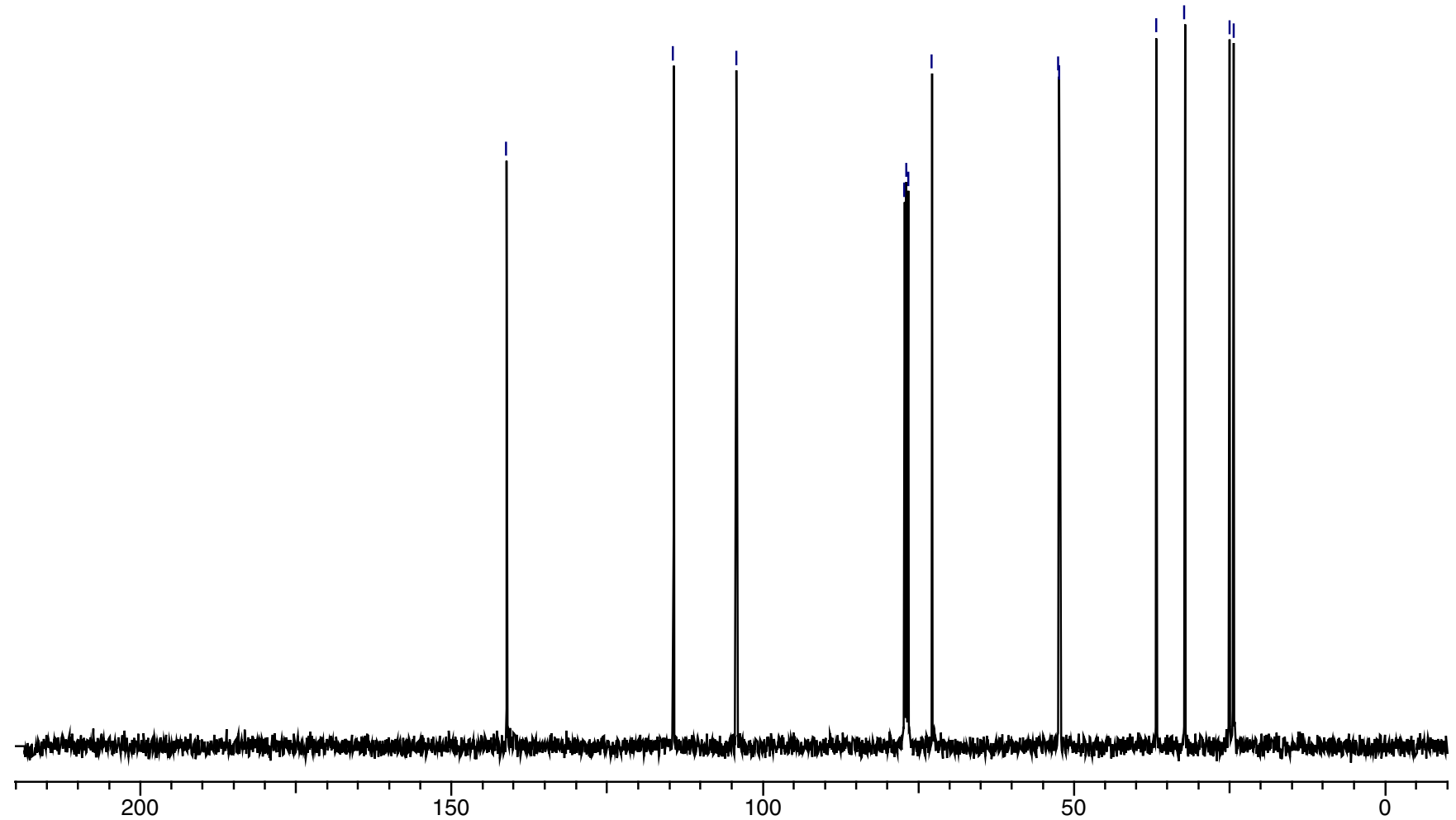




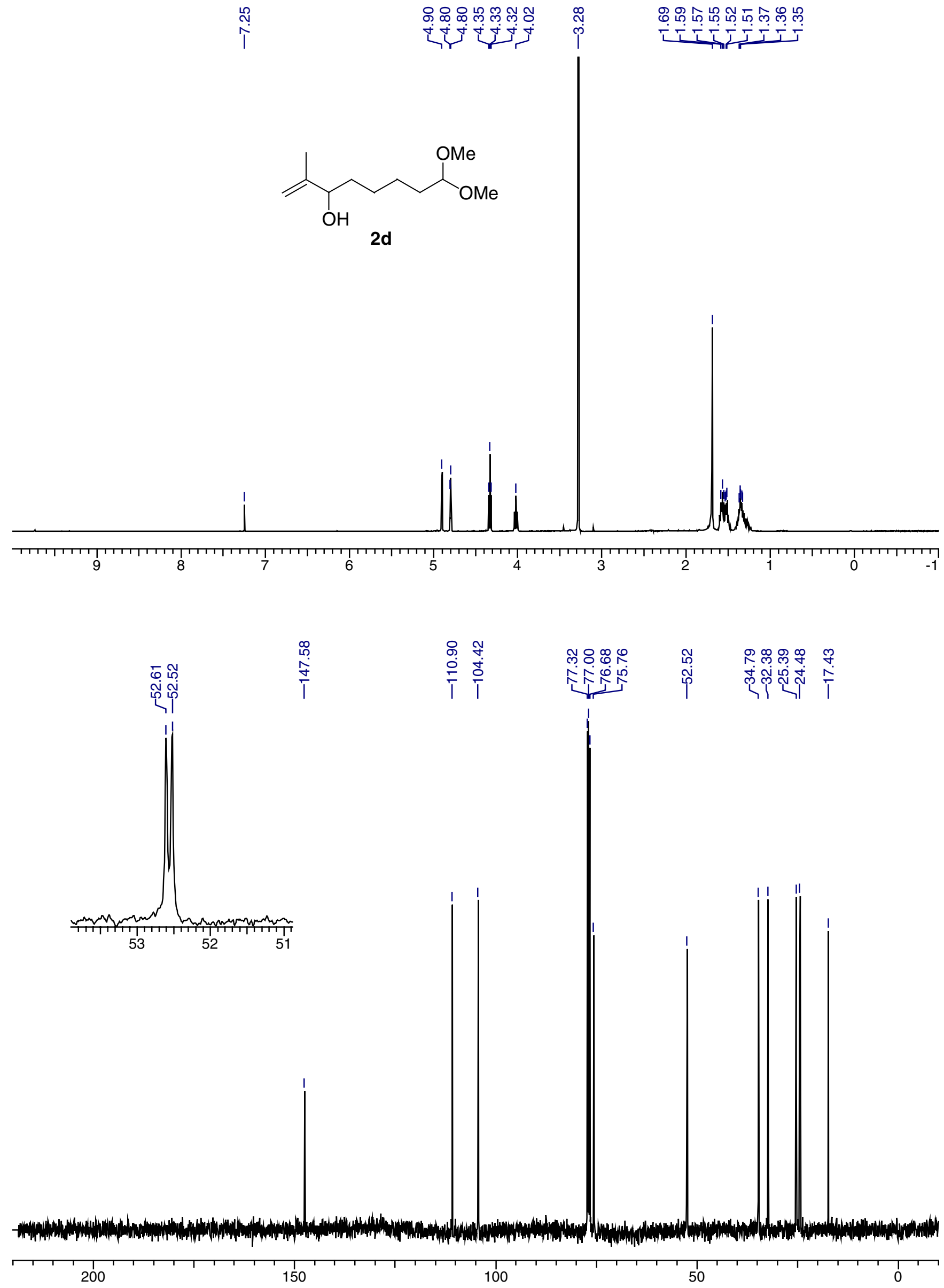




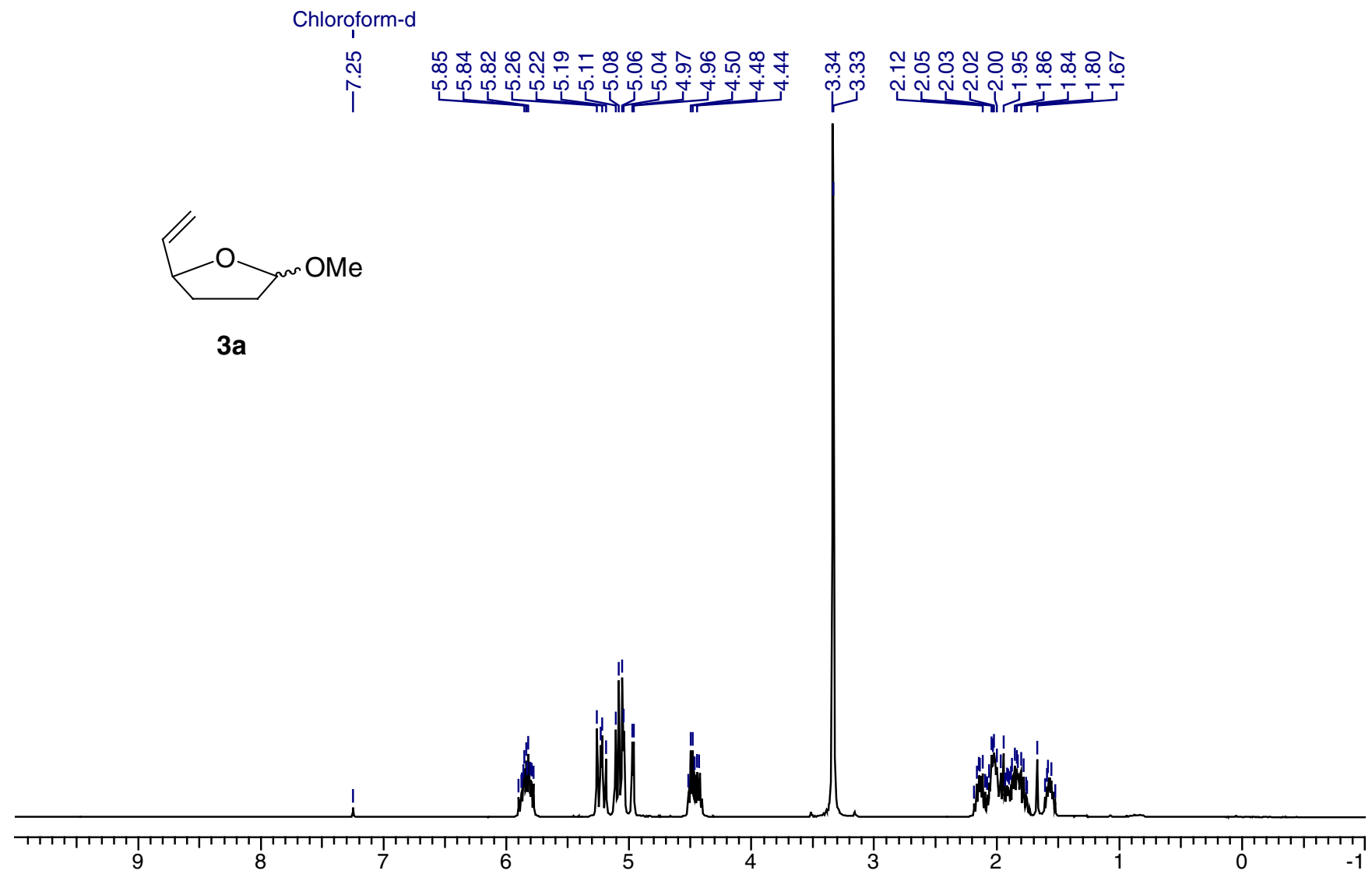

$\stackrel{\infty}{\infty}$

กิ

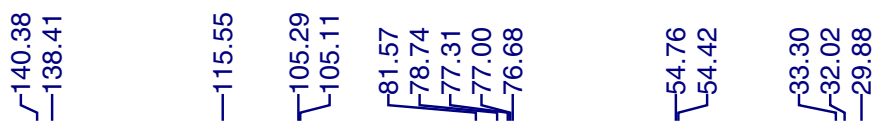

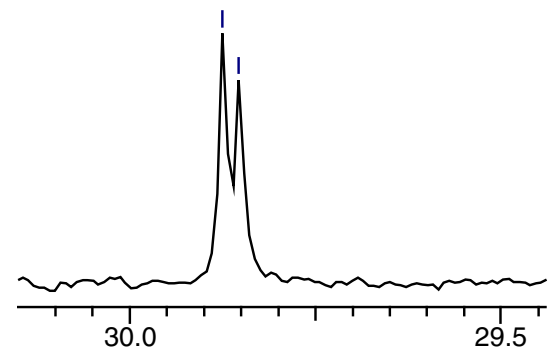




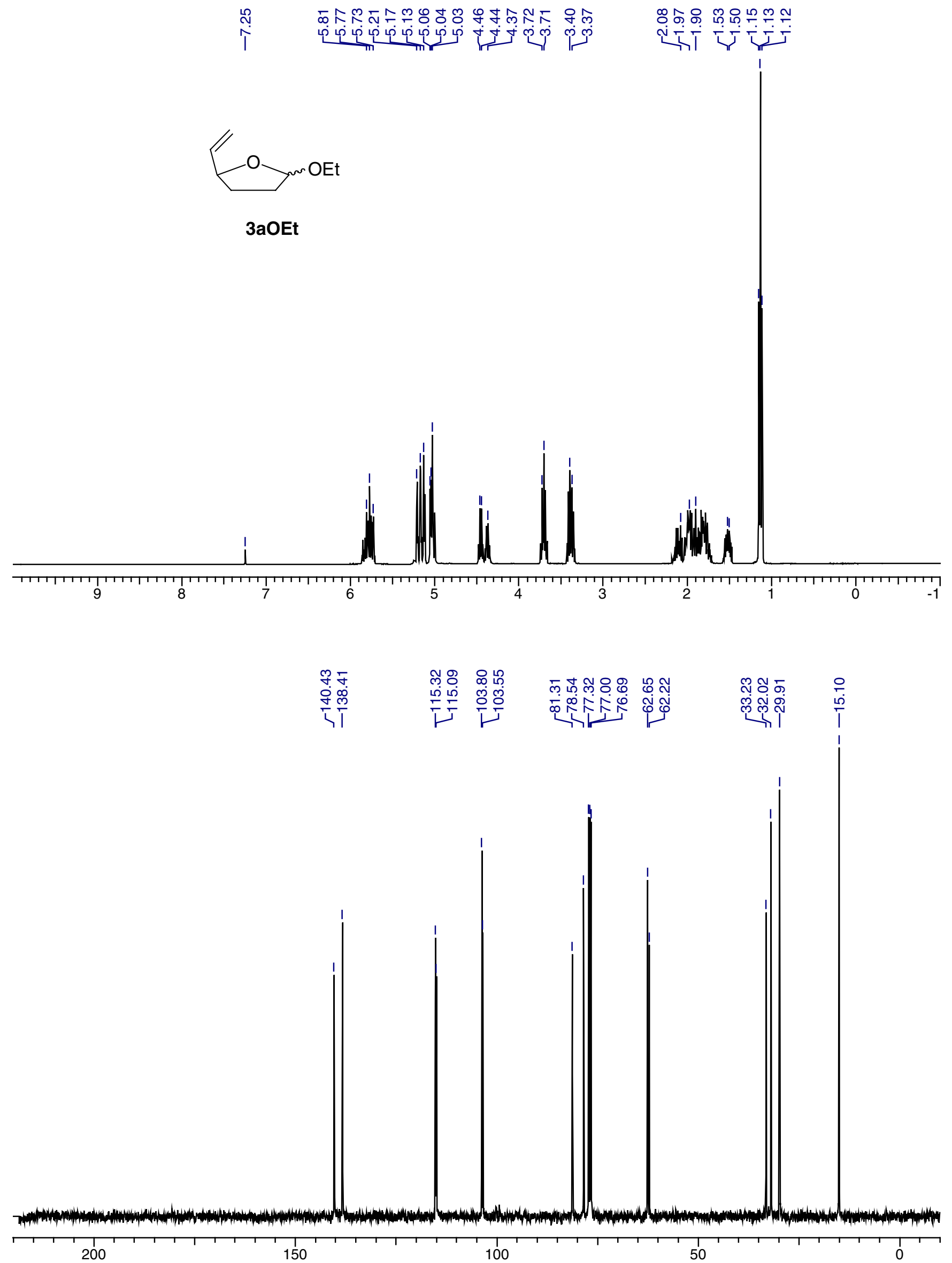




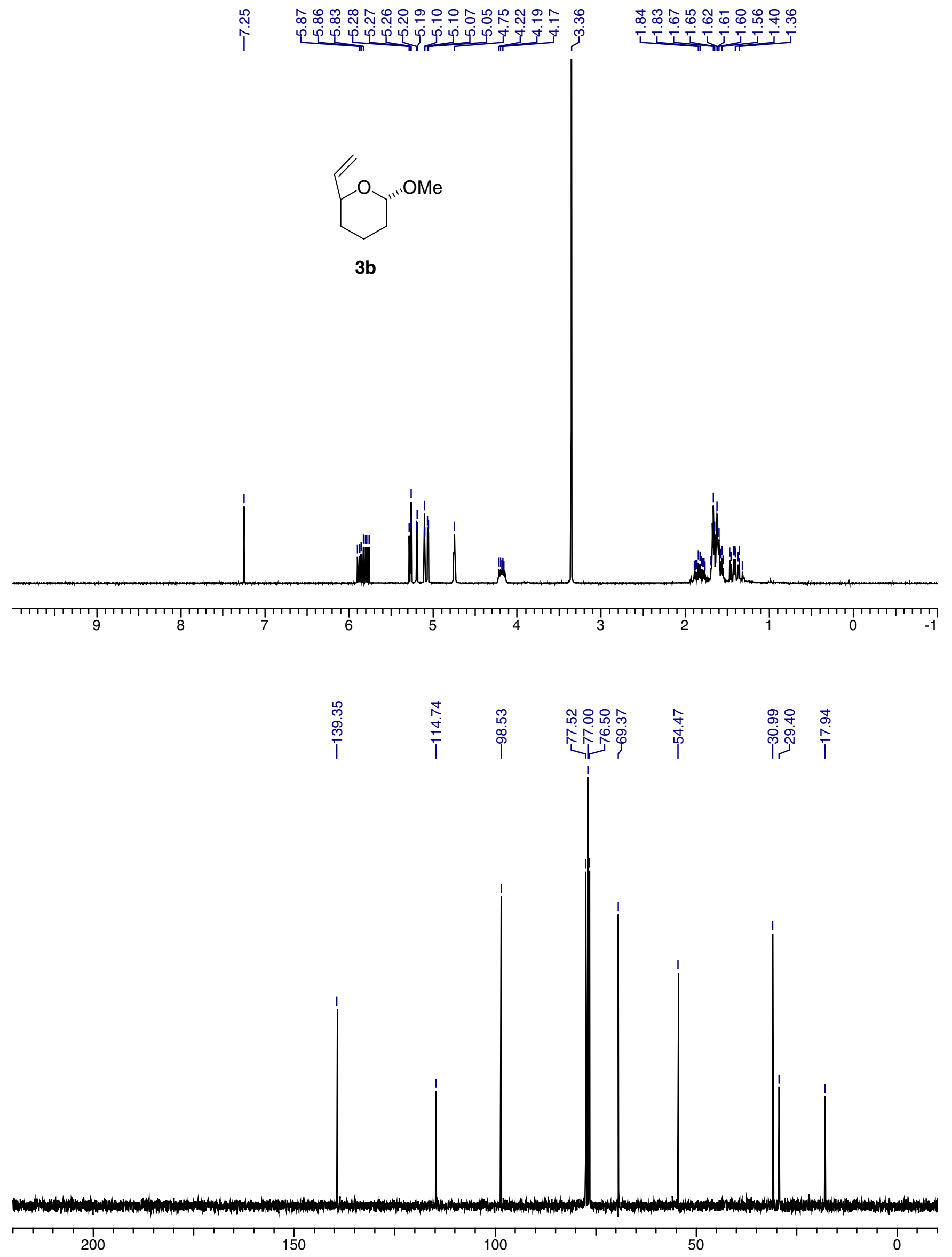




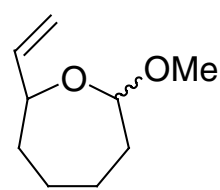

3c

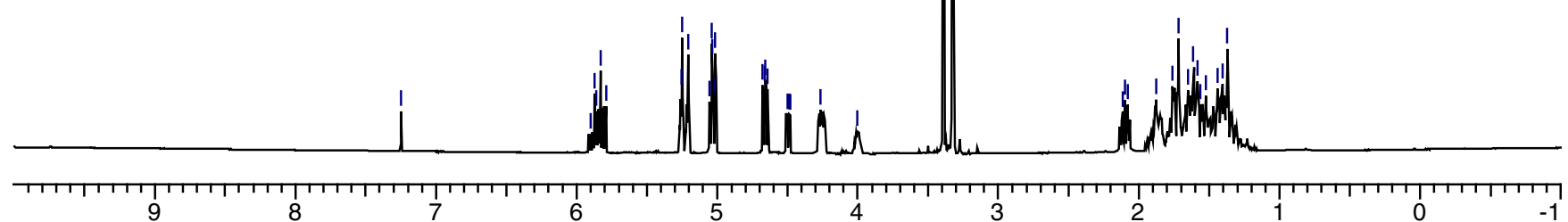

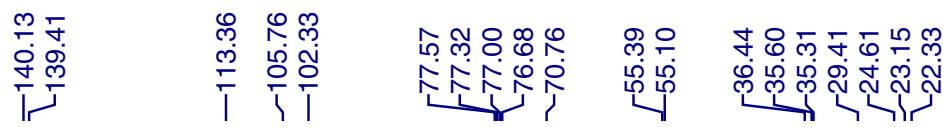

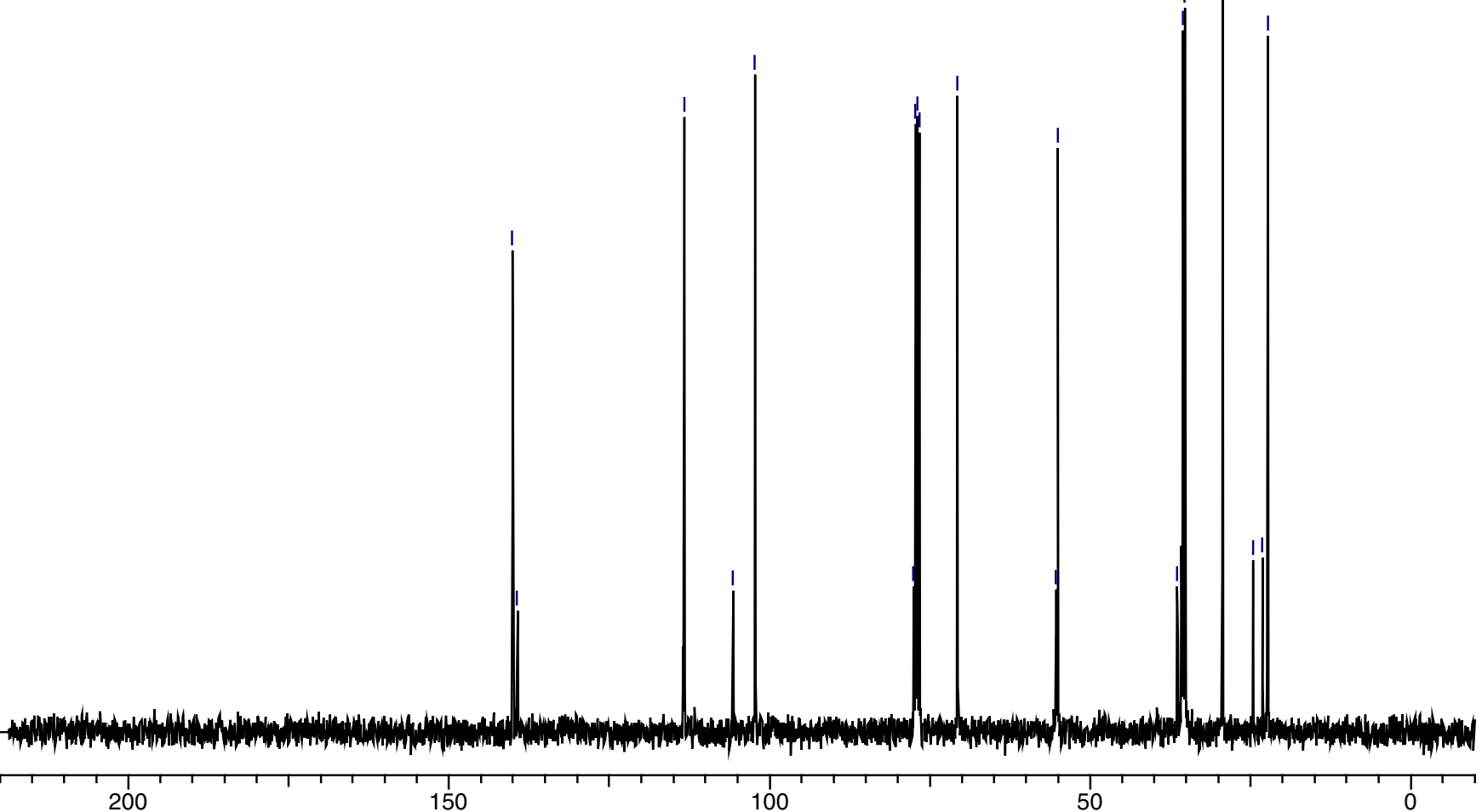



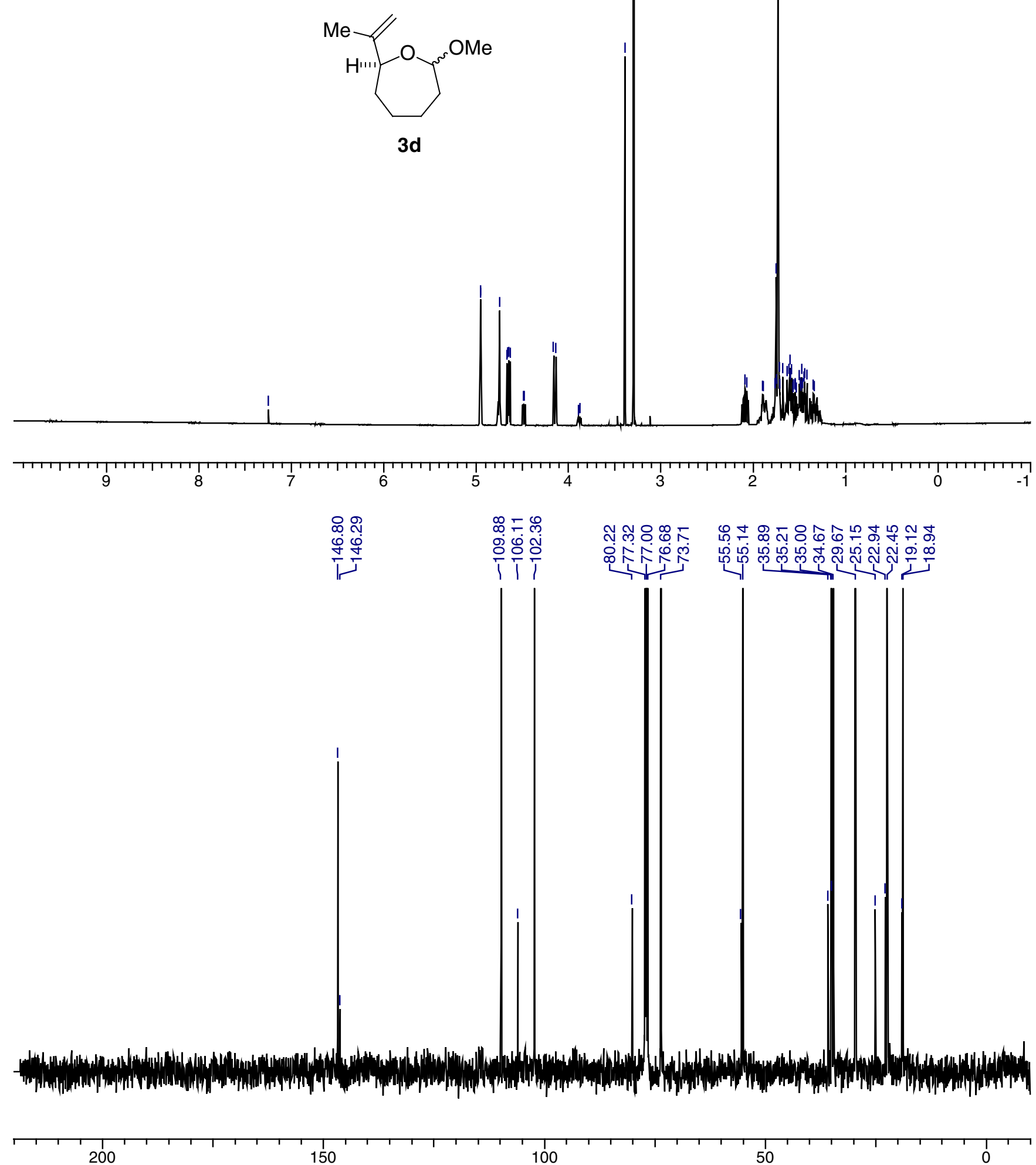


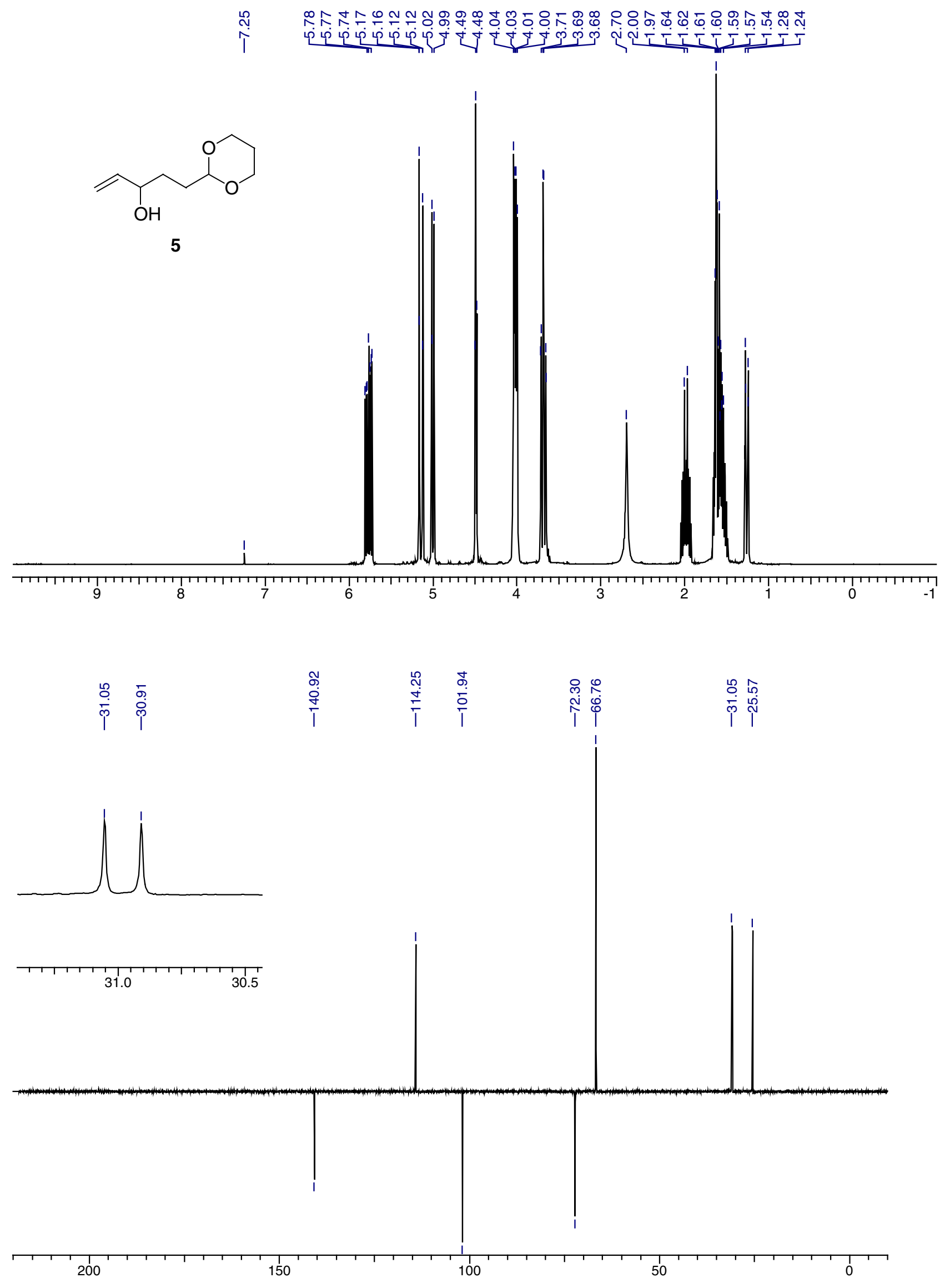


$7 a$

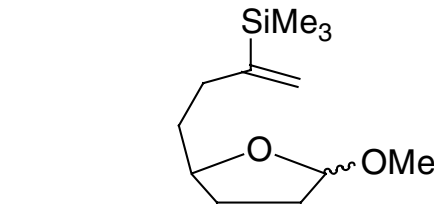

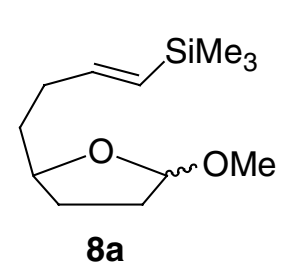

$8 a$

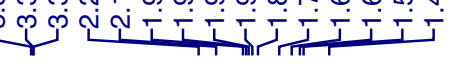

,
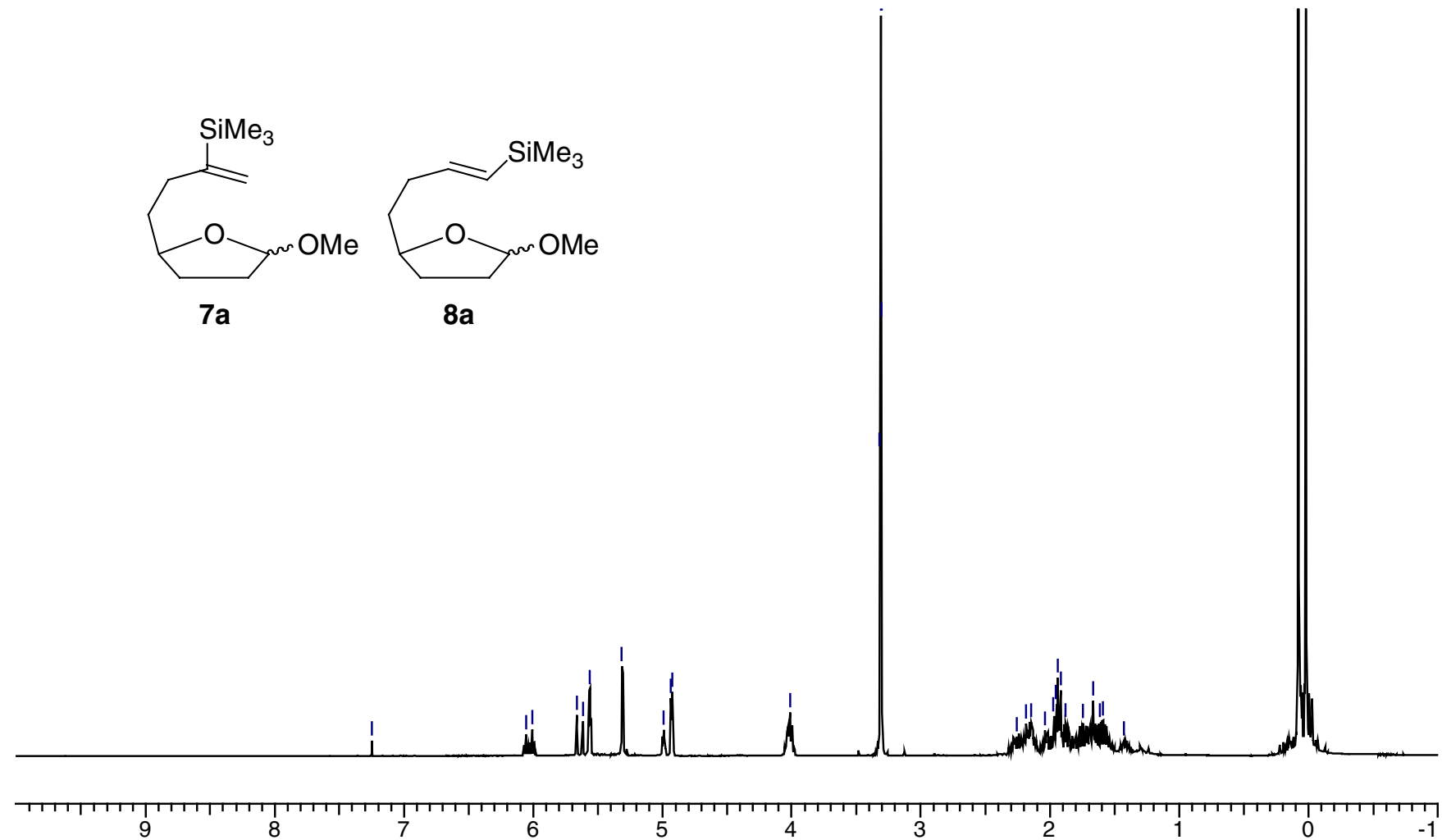

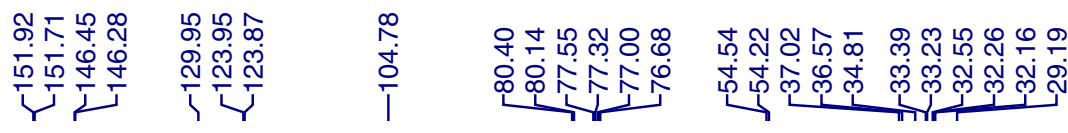

i̊

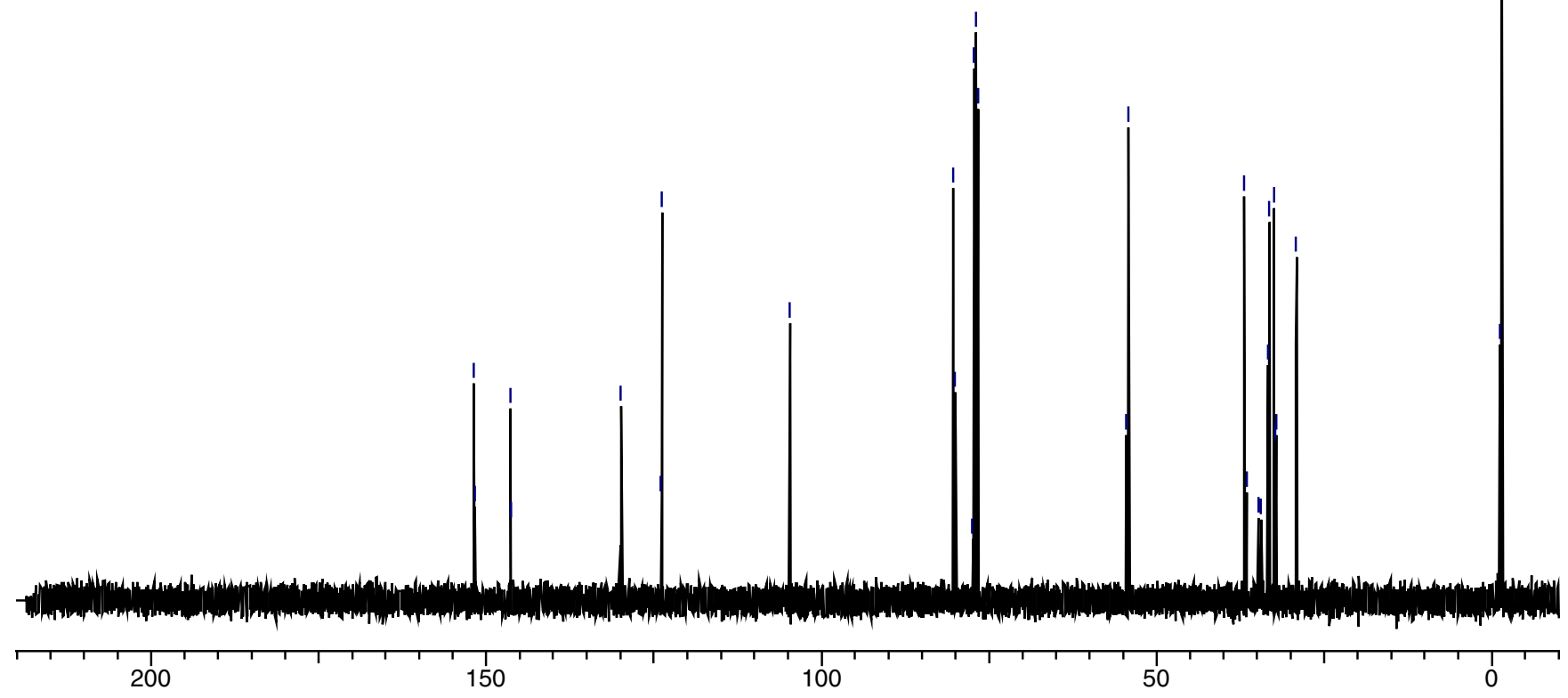




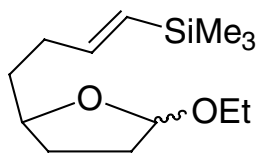

8aOEt

one diastereomer
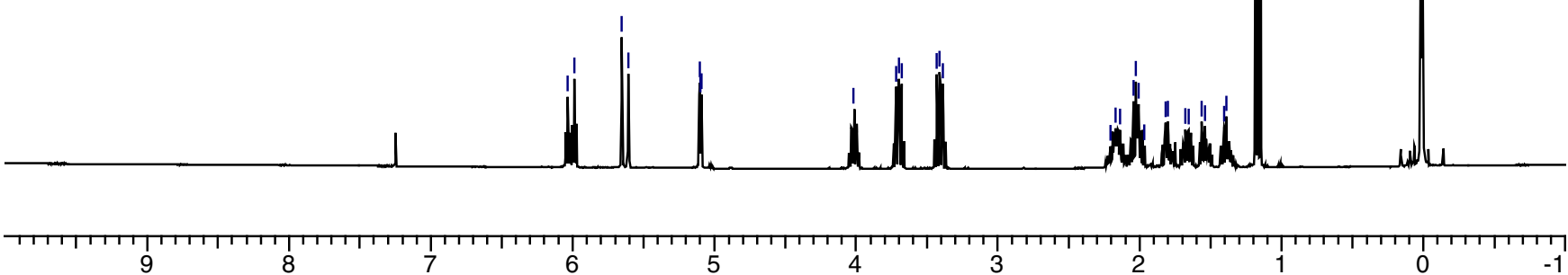

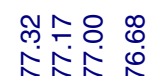

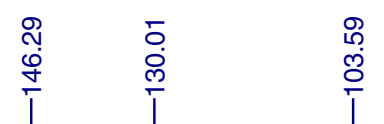

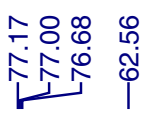

운소

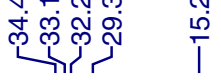

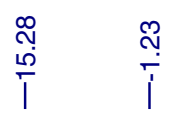

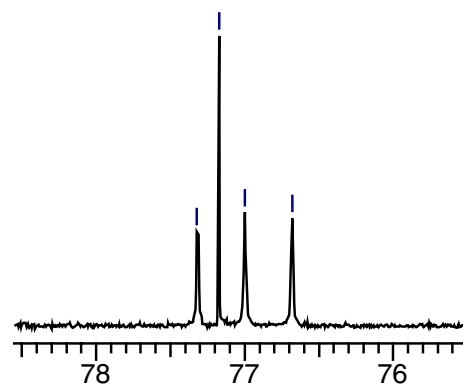

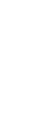


<smiles>C=C(C)[C@H]1CCC[C@@H]2CCC(=O)O[C@H]21</smiles>

7b

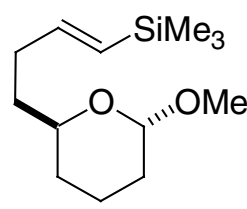

$8 b$

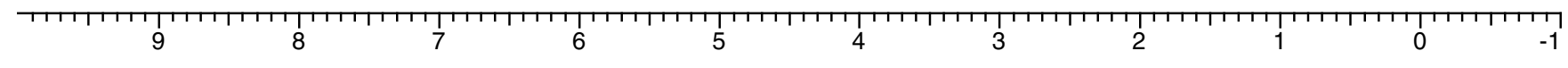

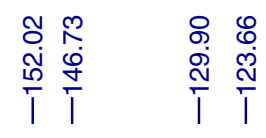

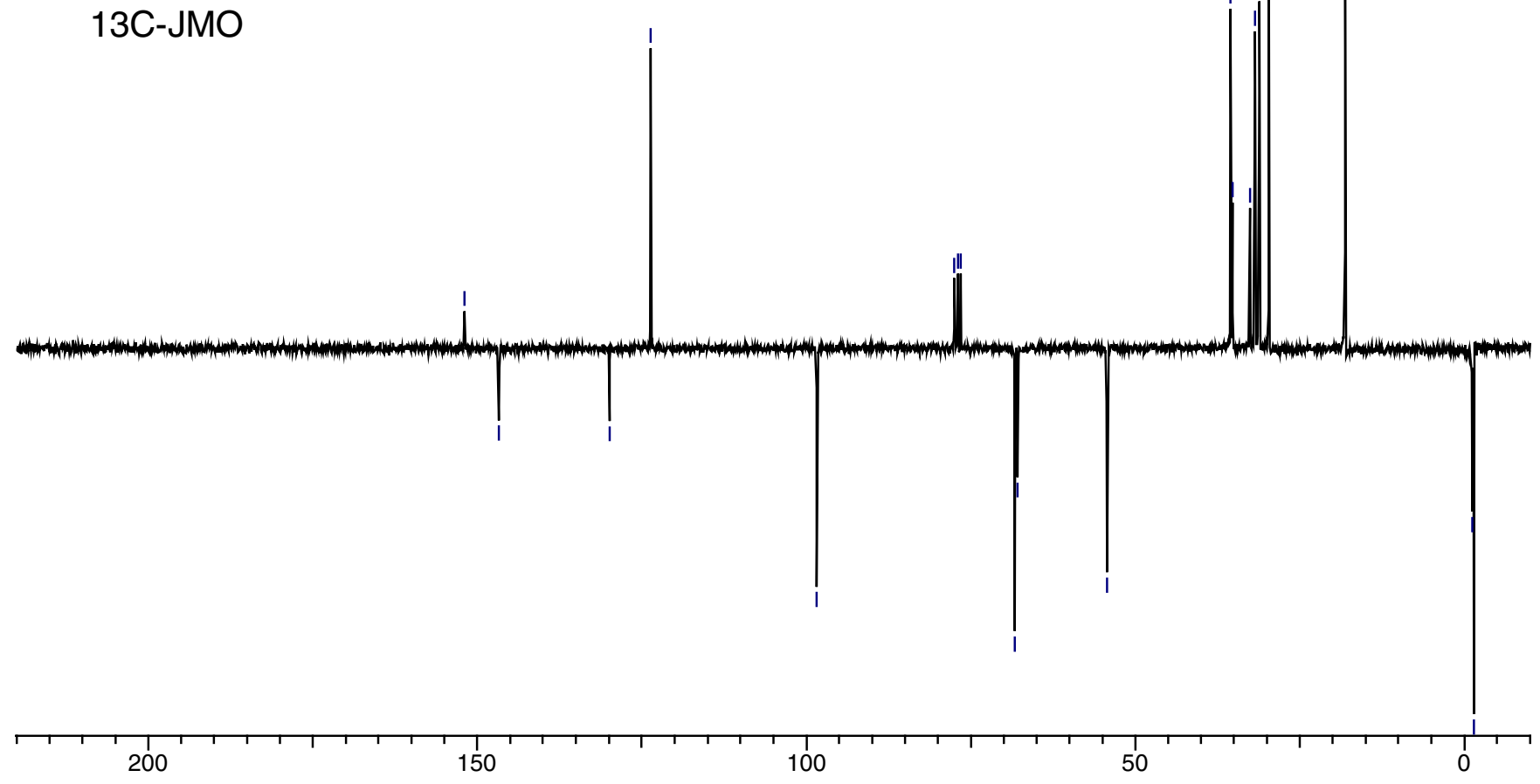




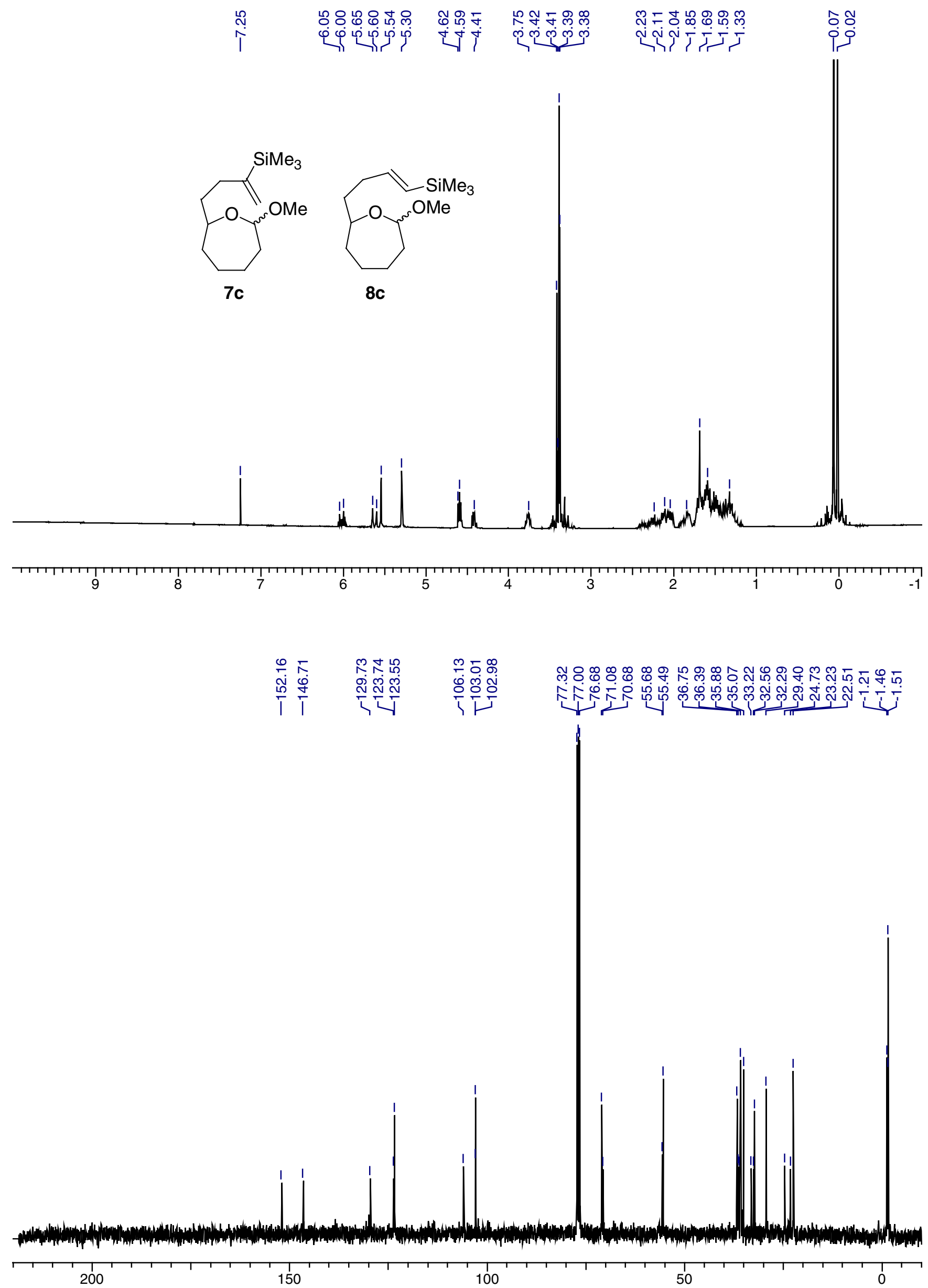




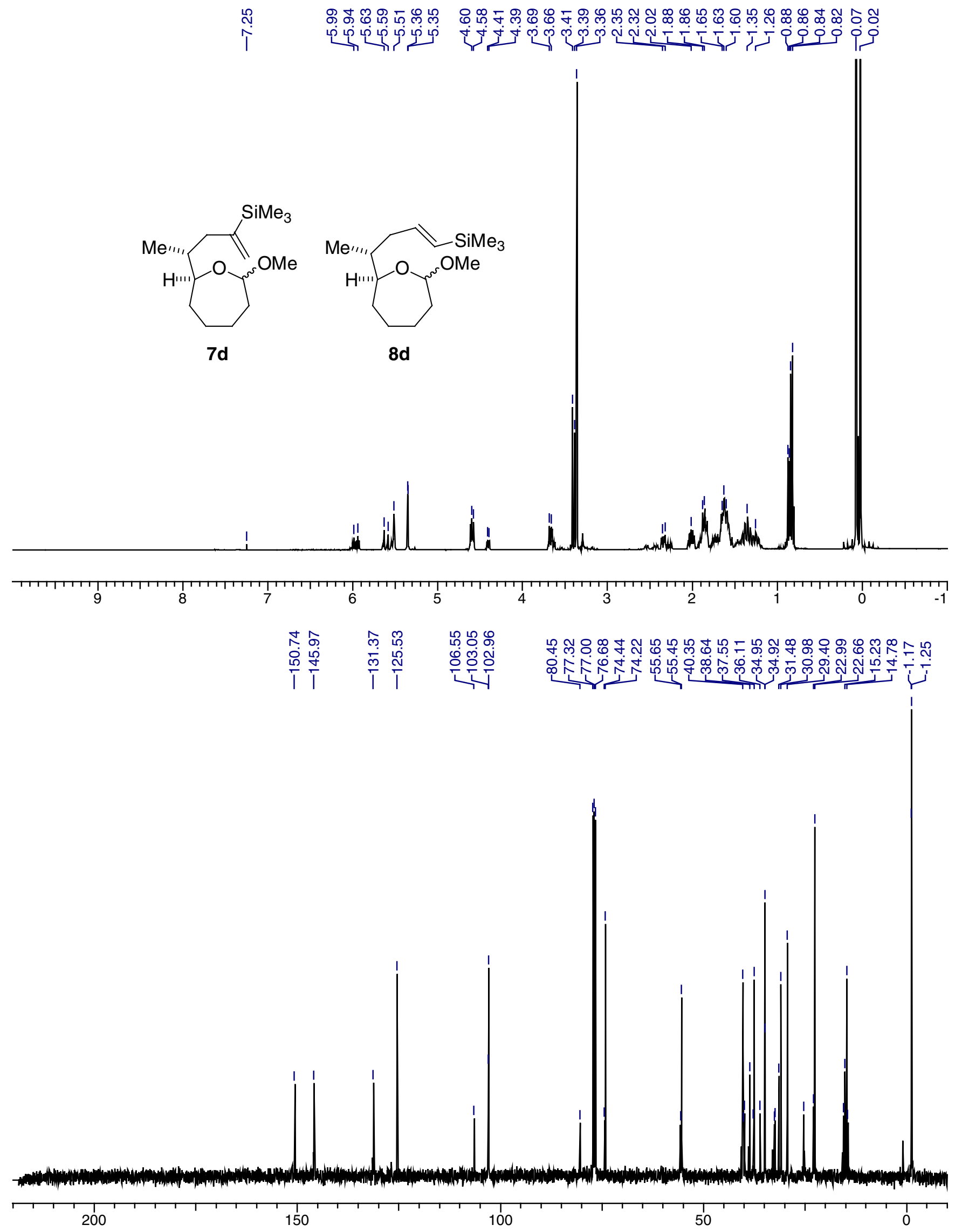




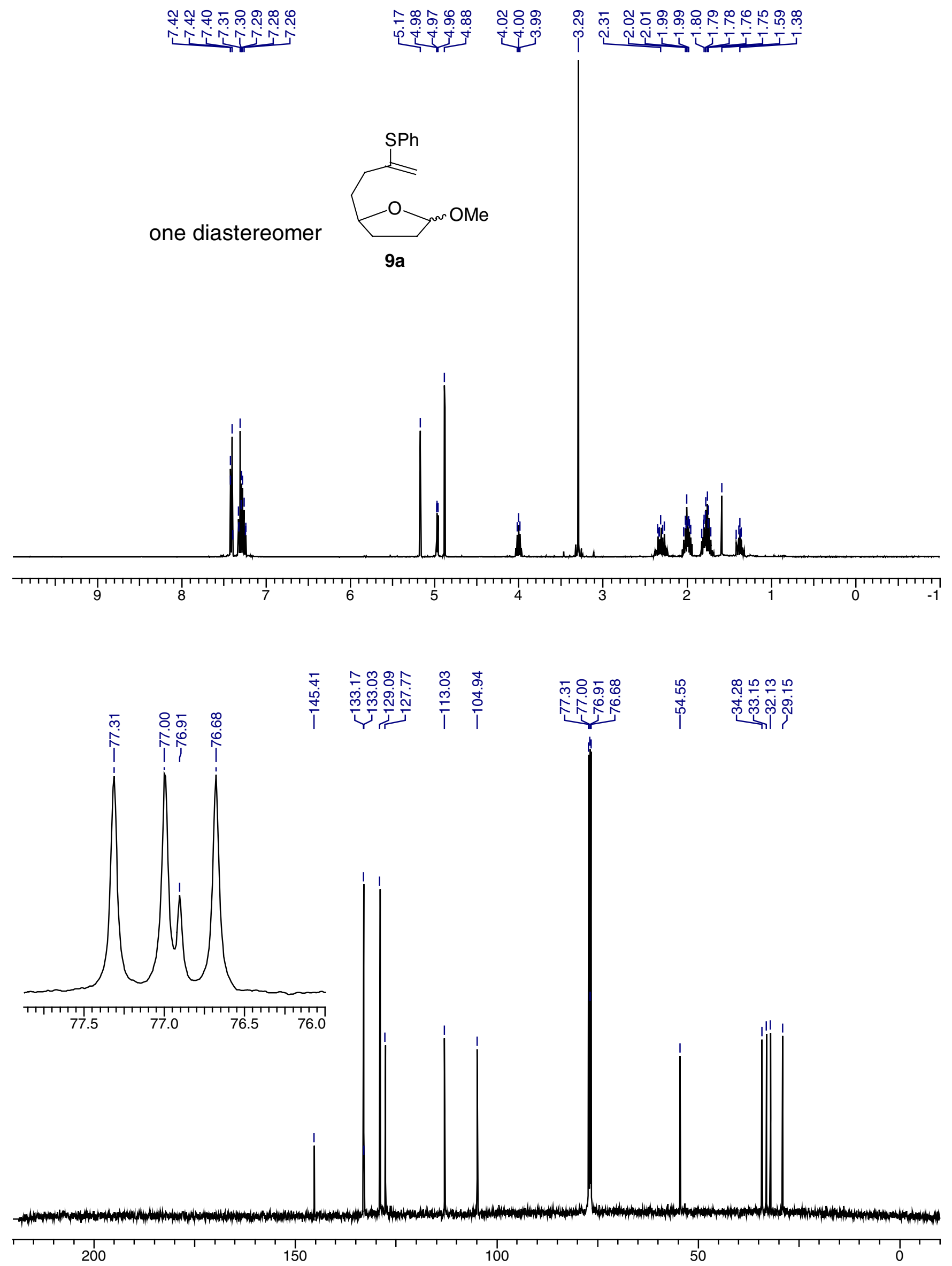




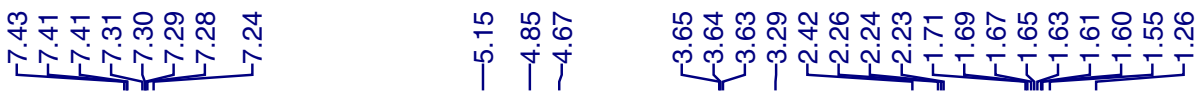

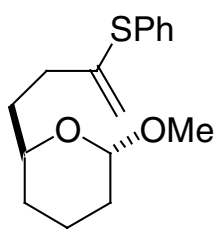

$9 b$

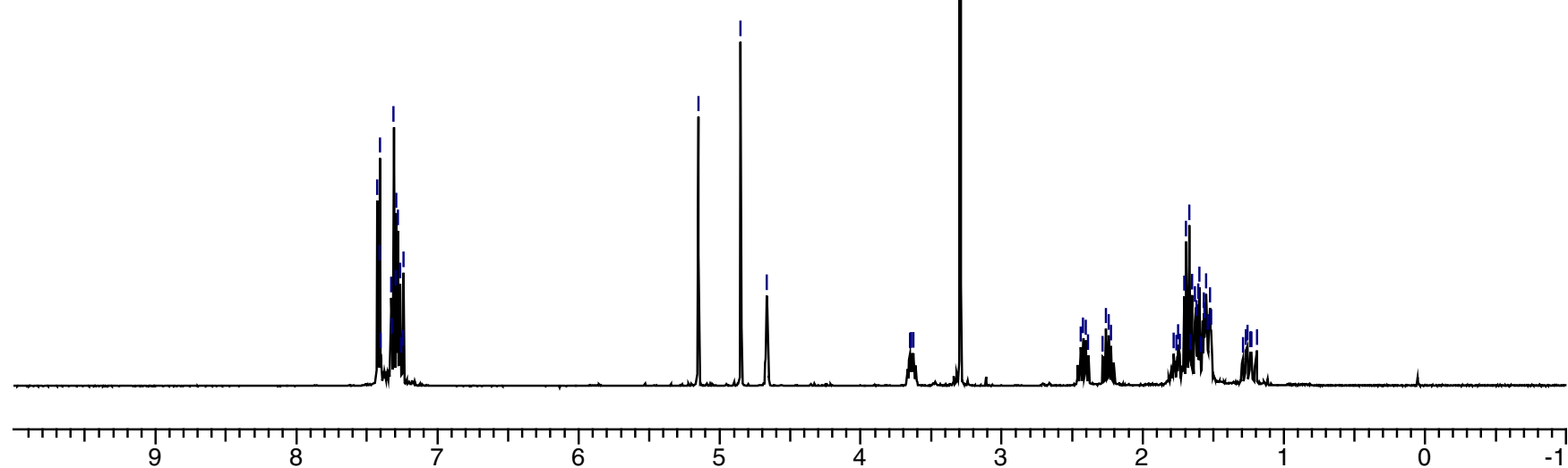

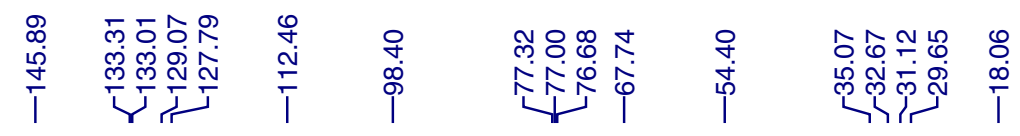

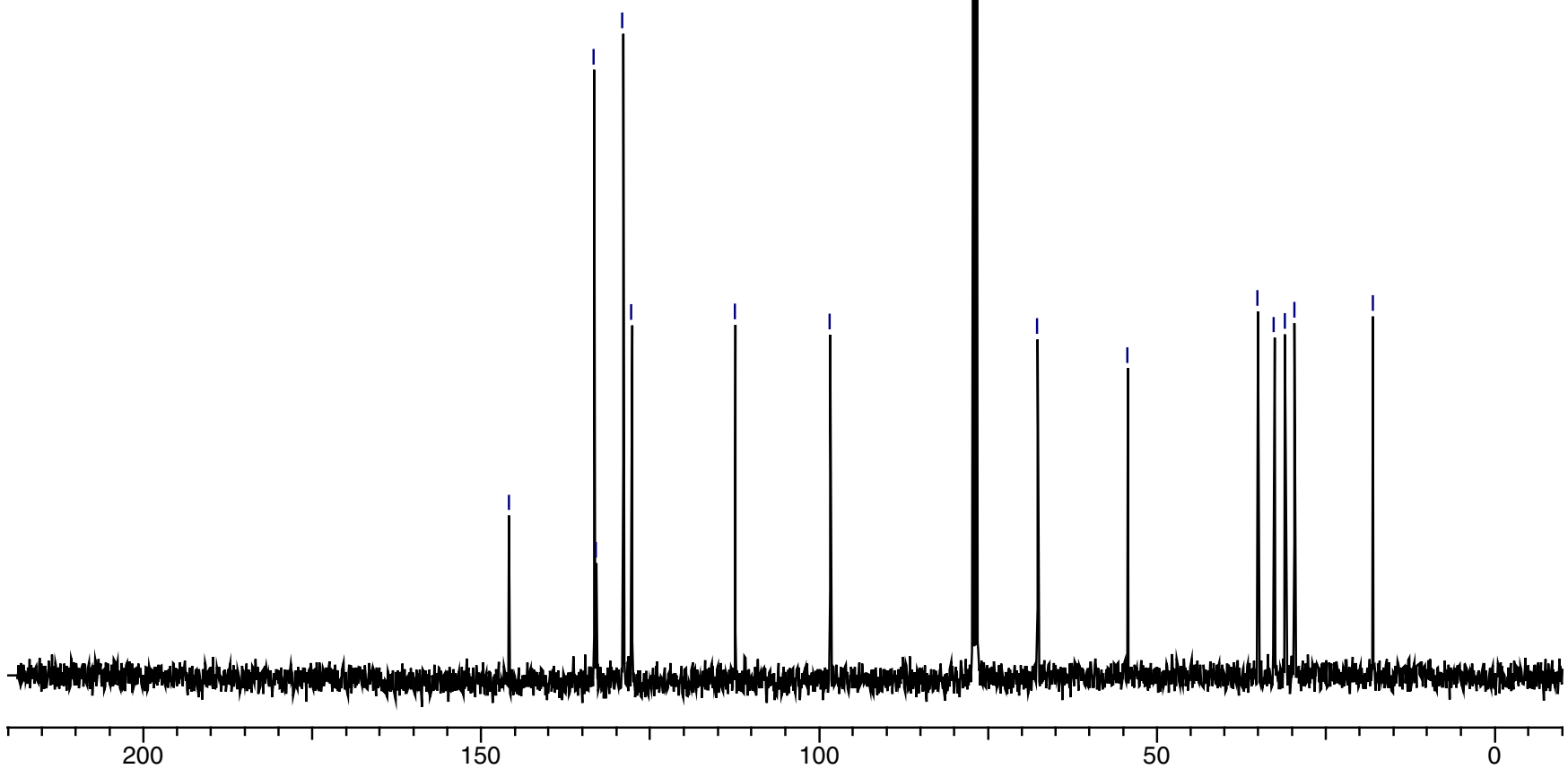




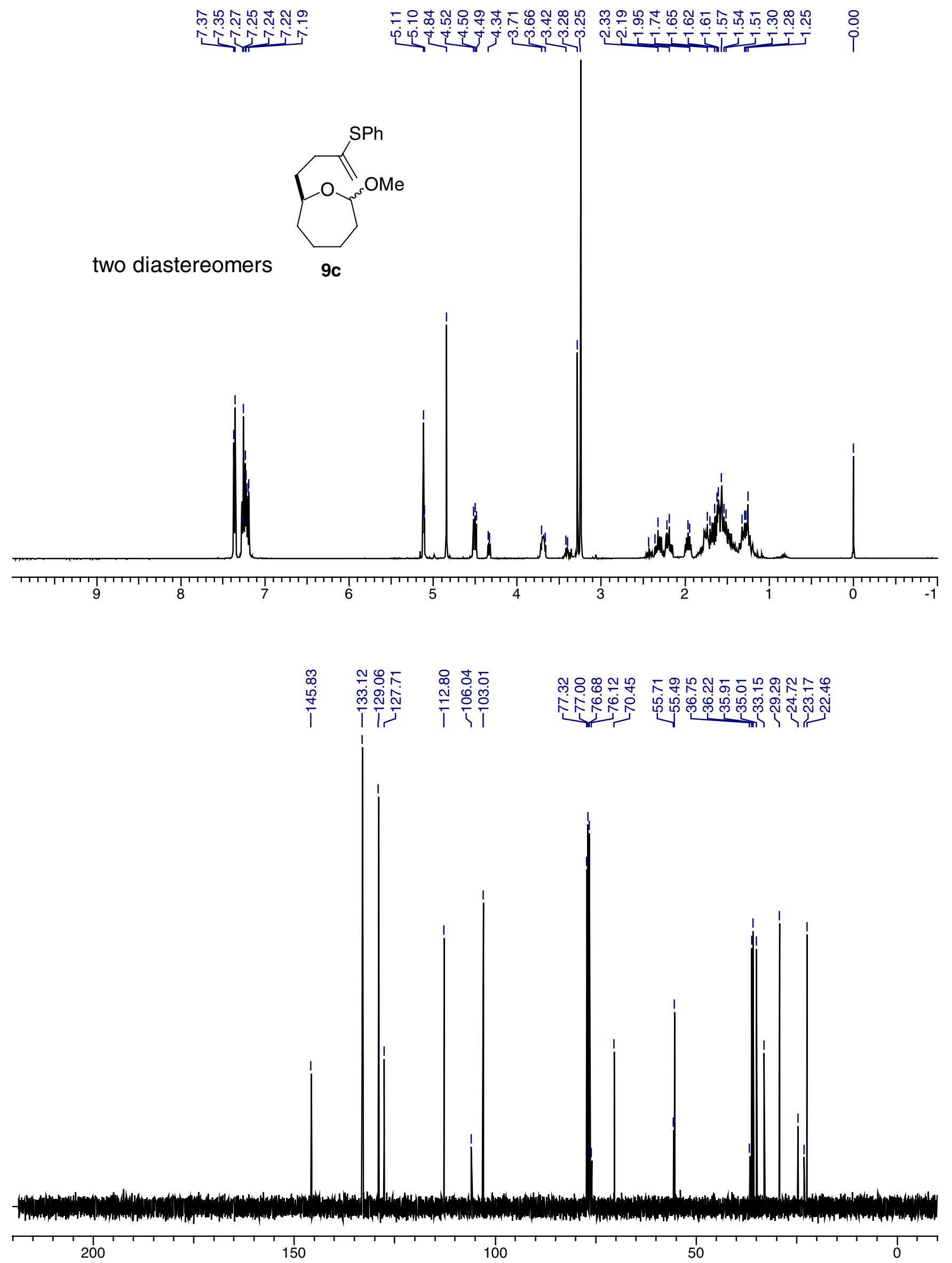




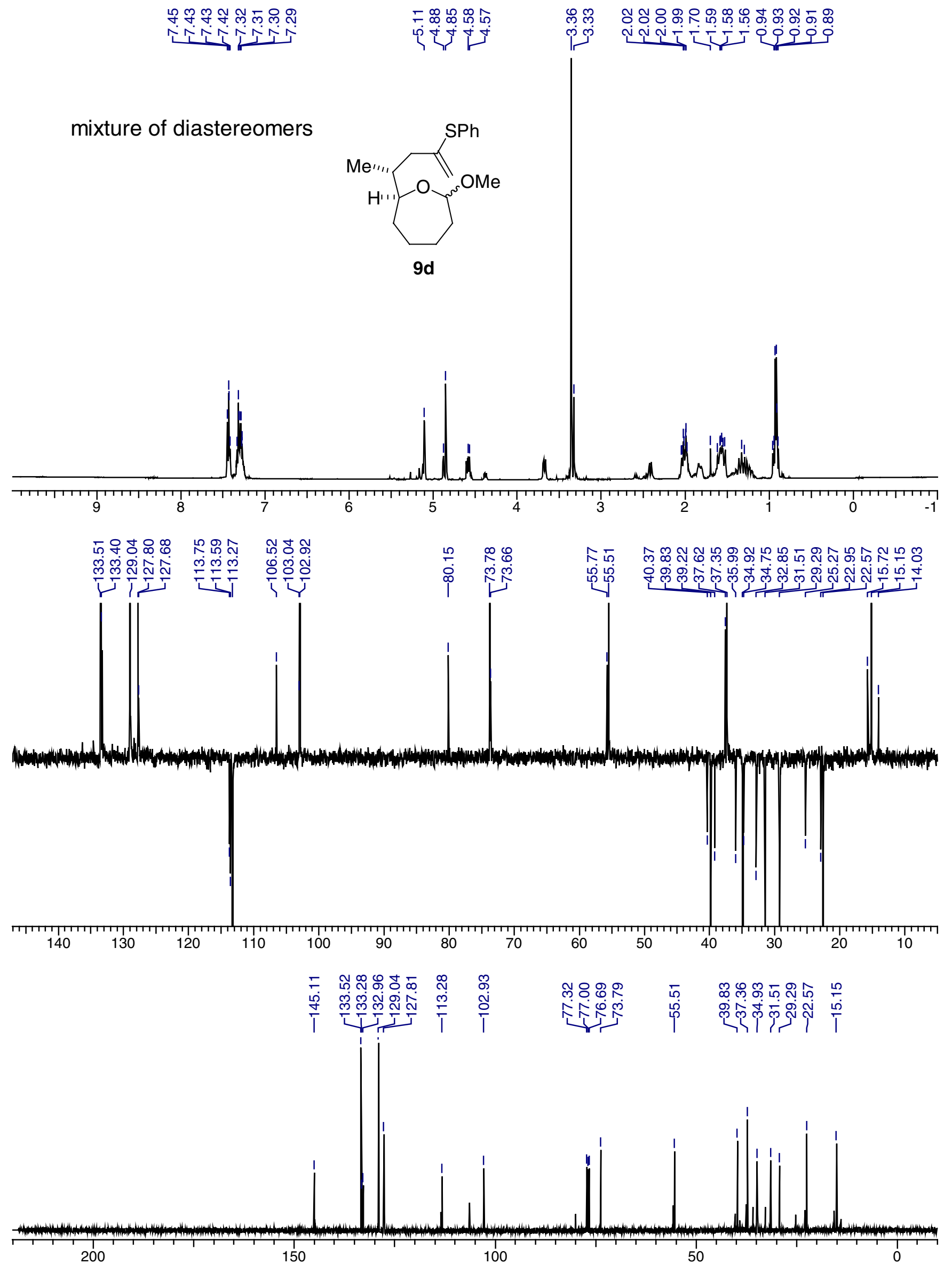




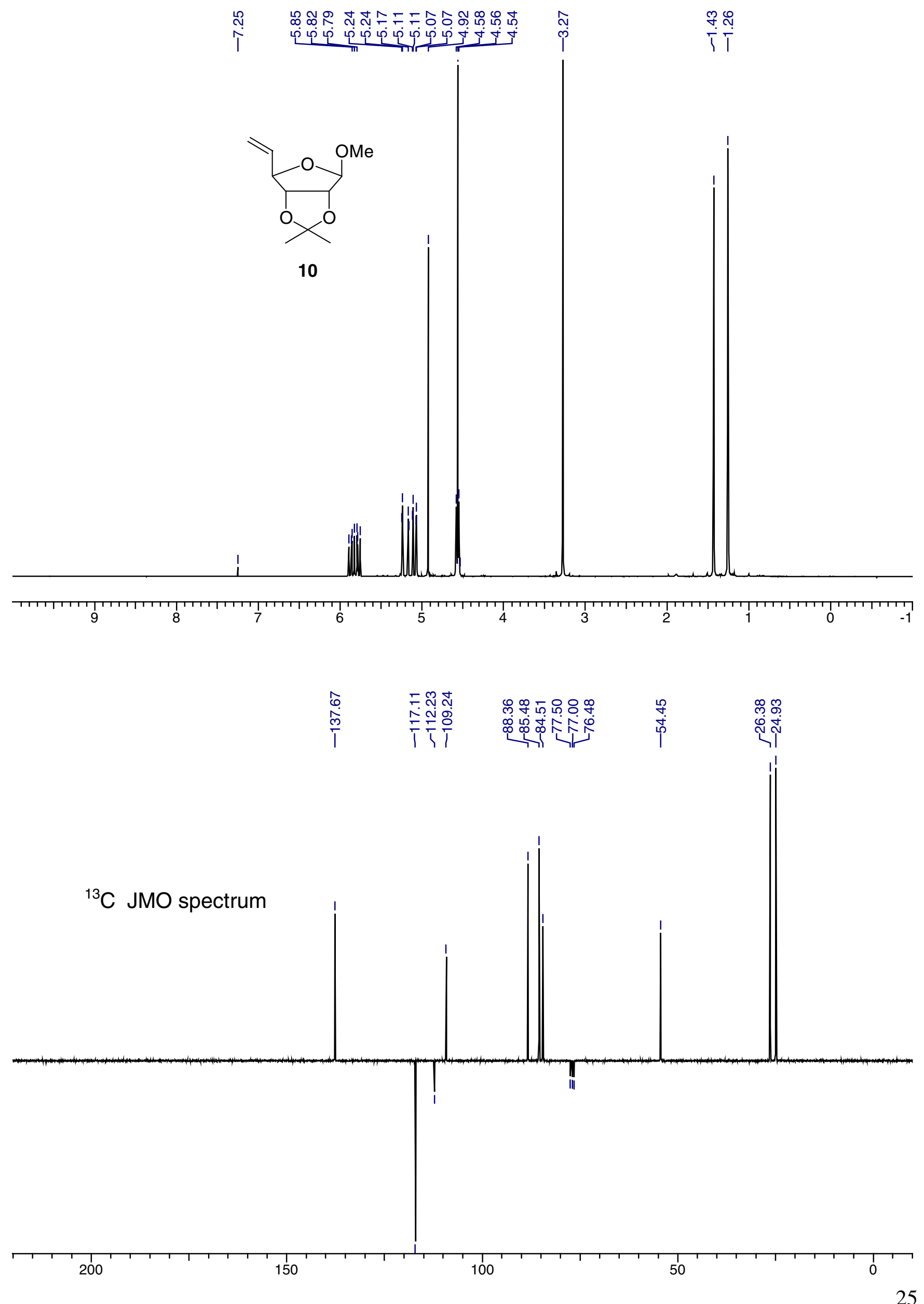


<smiles>C=C(C)CCC1OC2OC(C)(C)OC1C2OC</smiles>

11

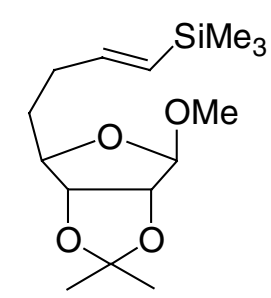

12

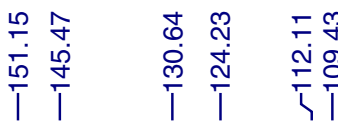

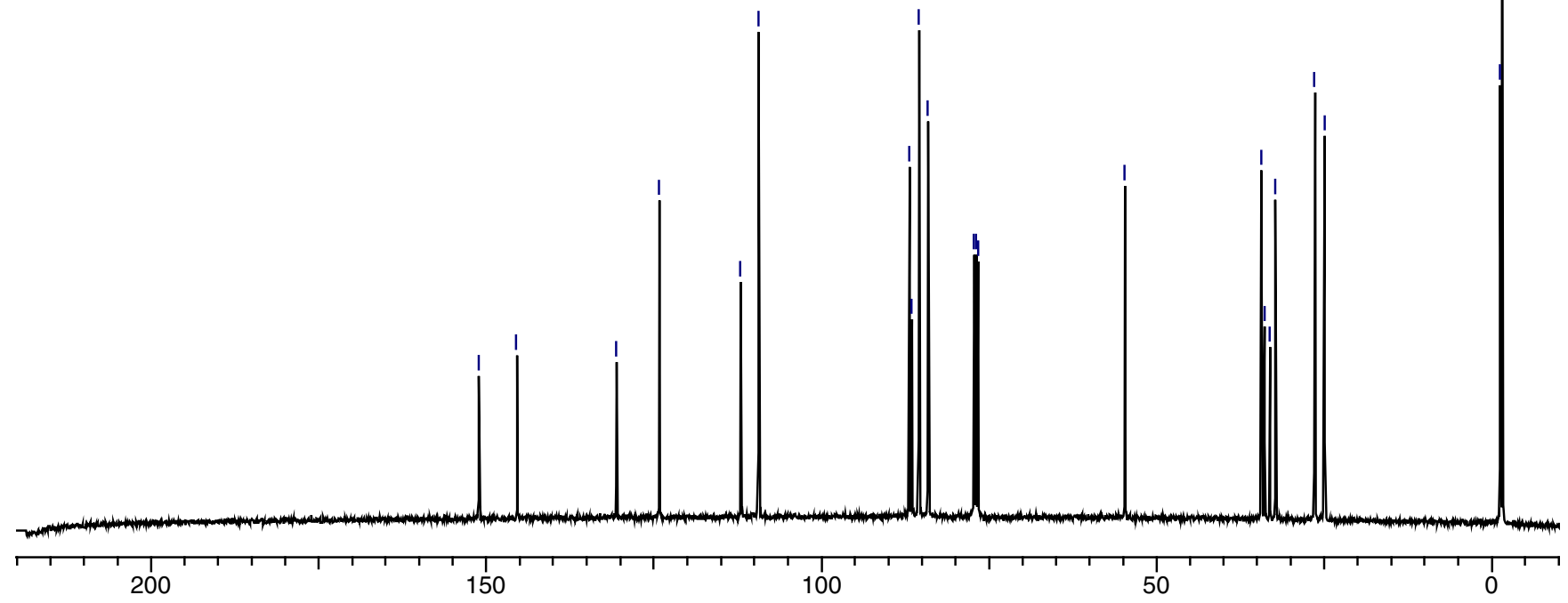



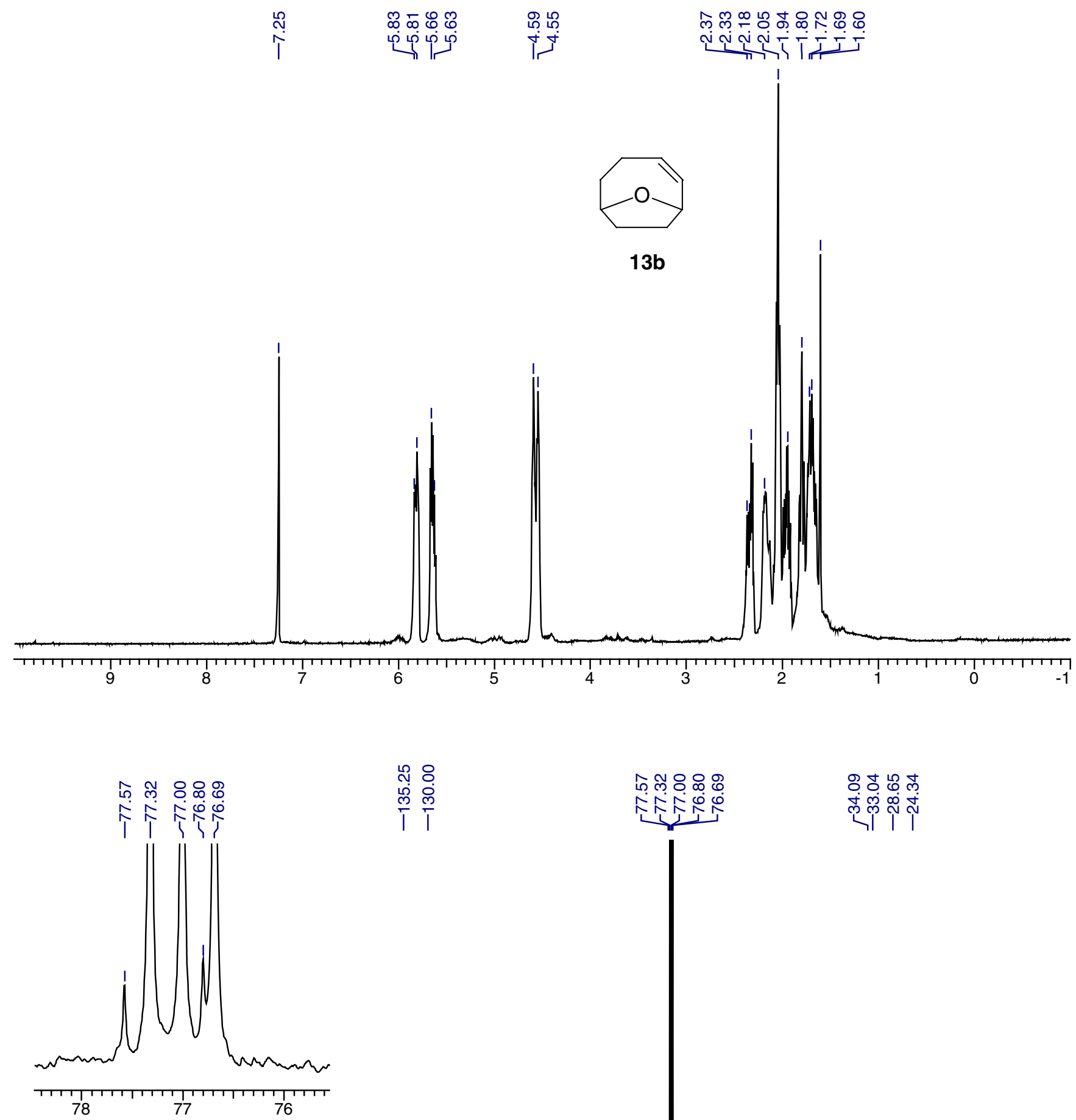

กุ 8

लिं

ำ 이요요

余令尔

○すั

लूं क्ञ

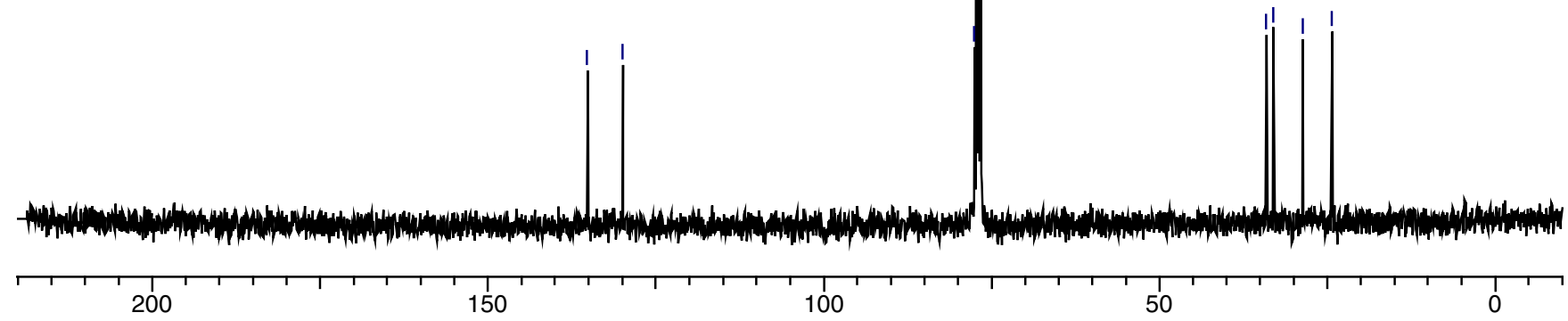



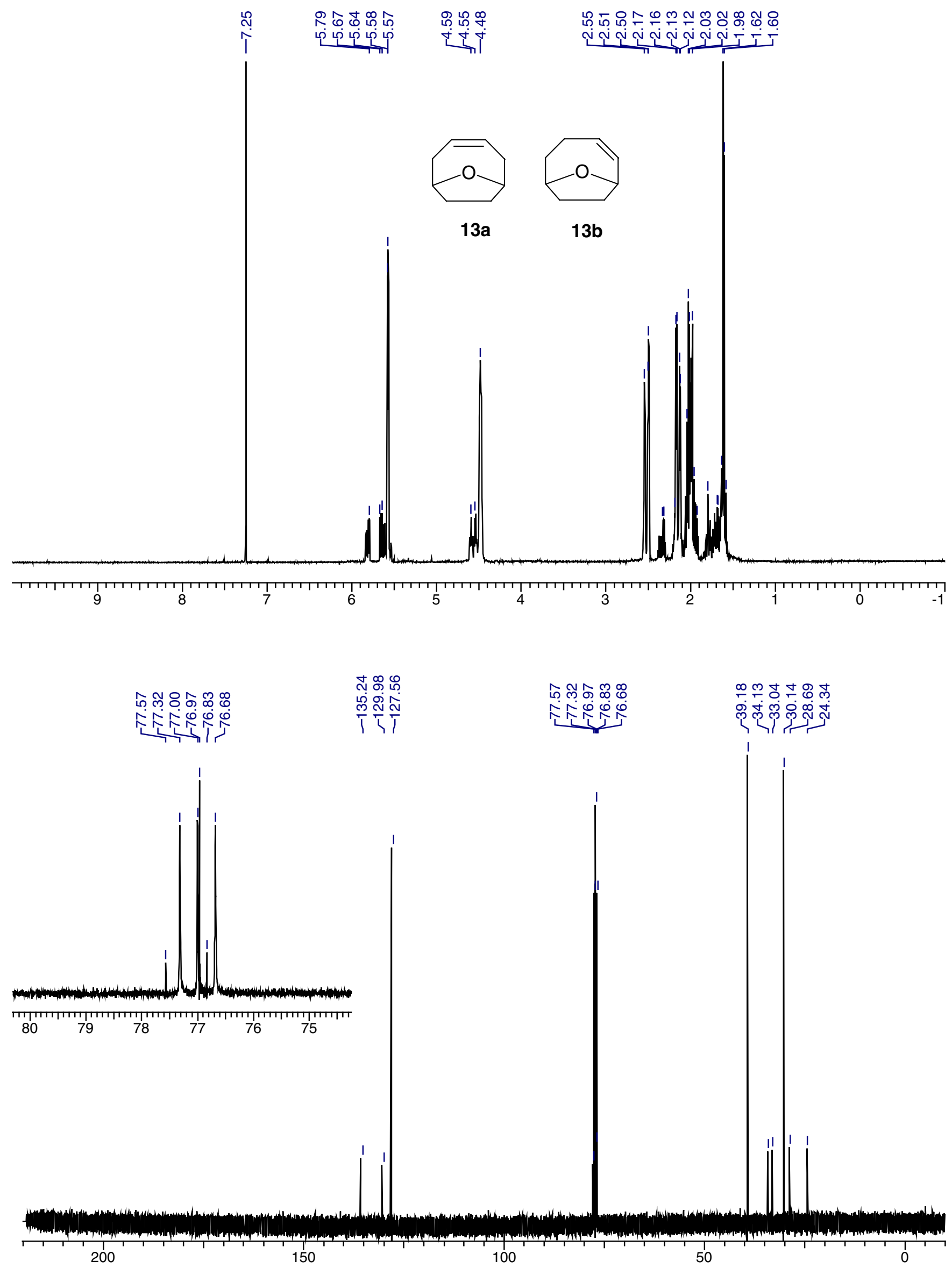
న़门

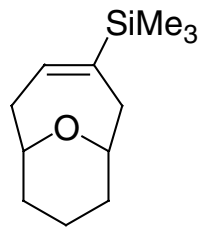

$14 a$

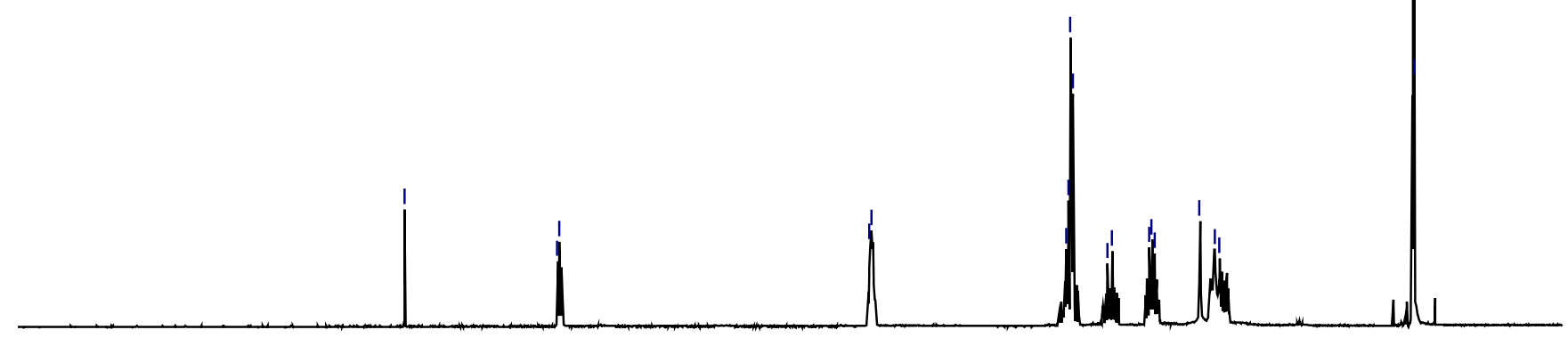

ภ ळ

พั่

ஸิ 웅ำ

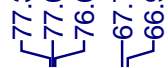

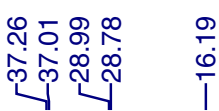

กั

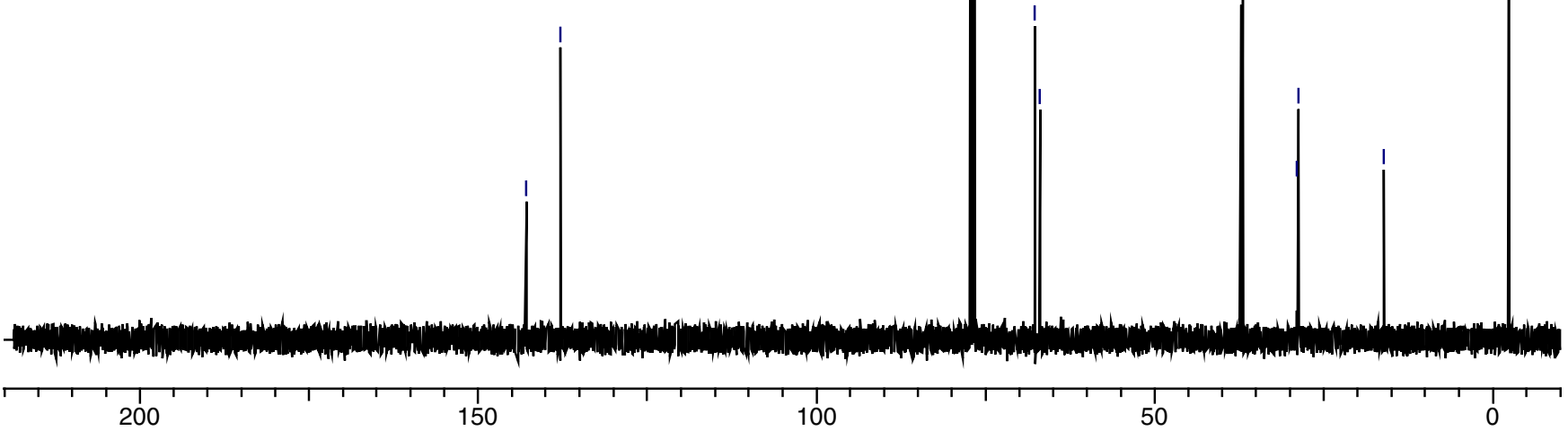



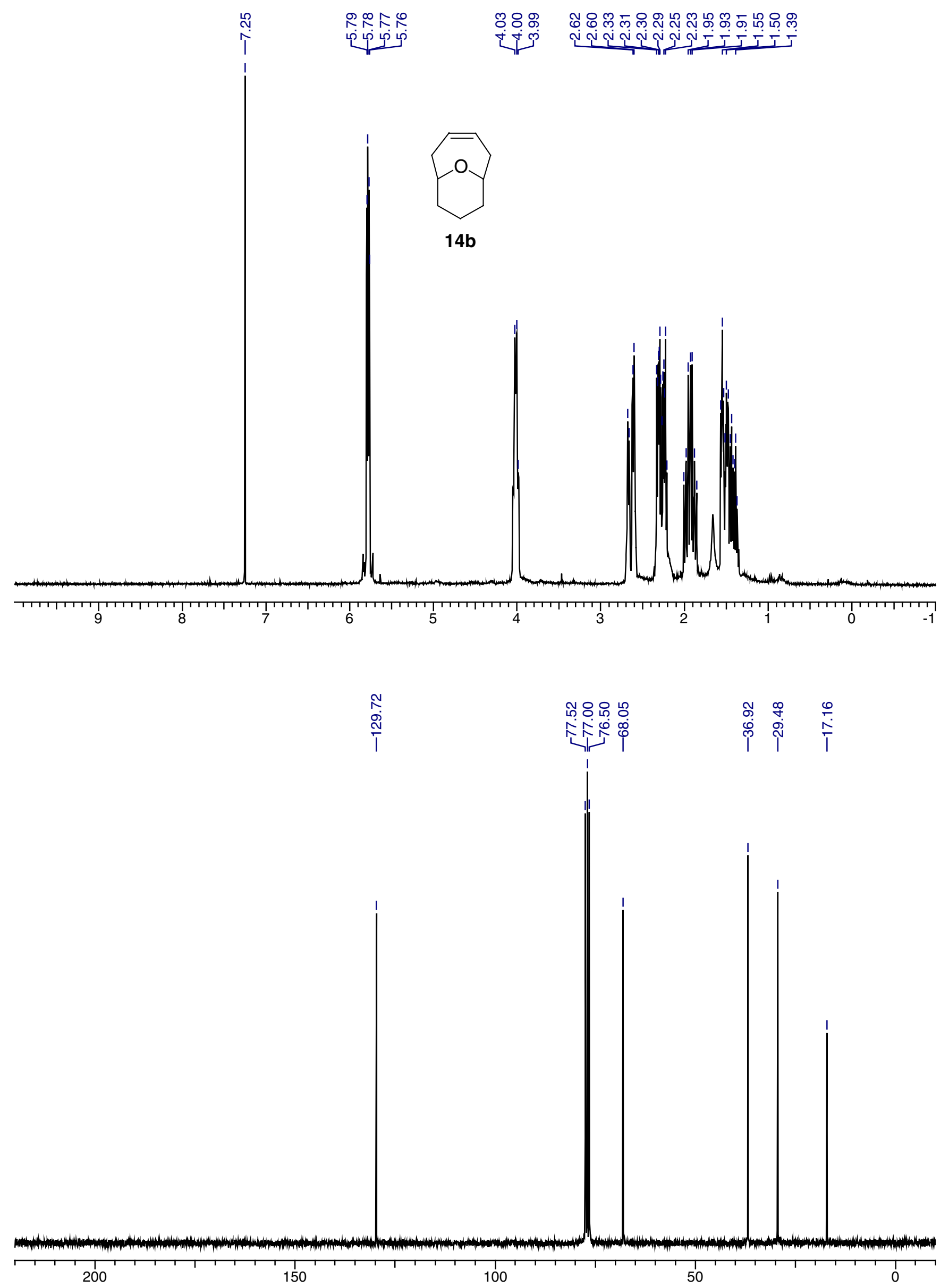

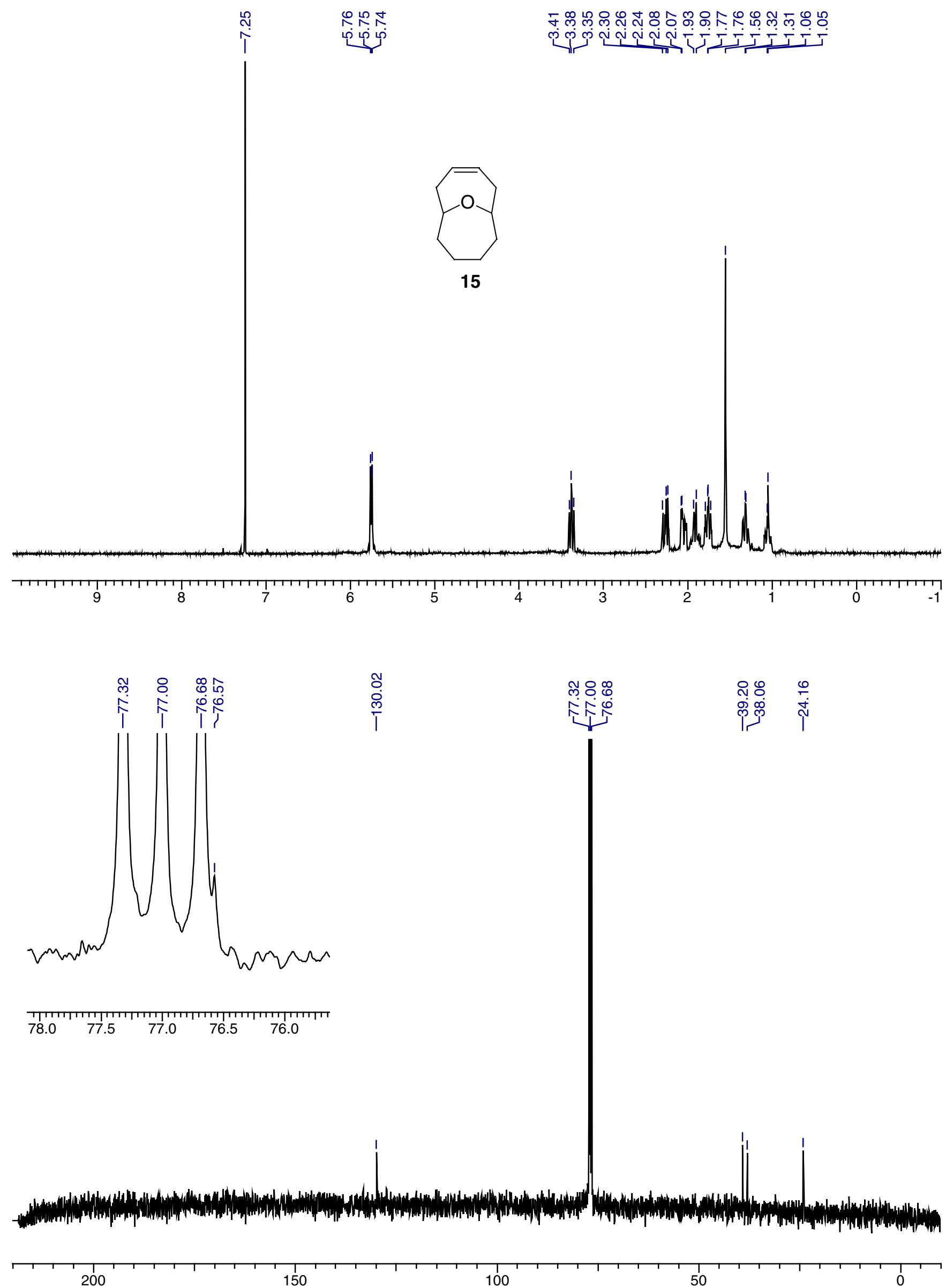

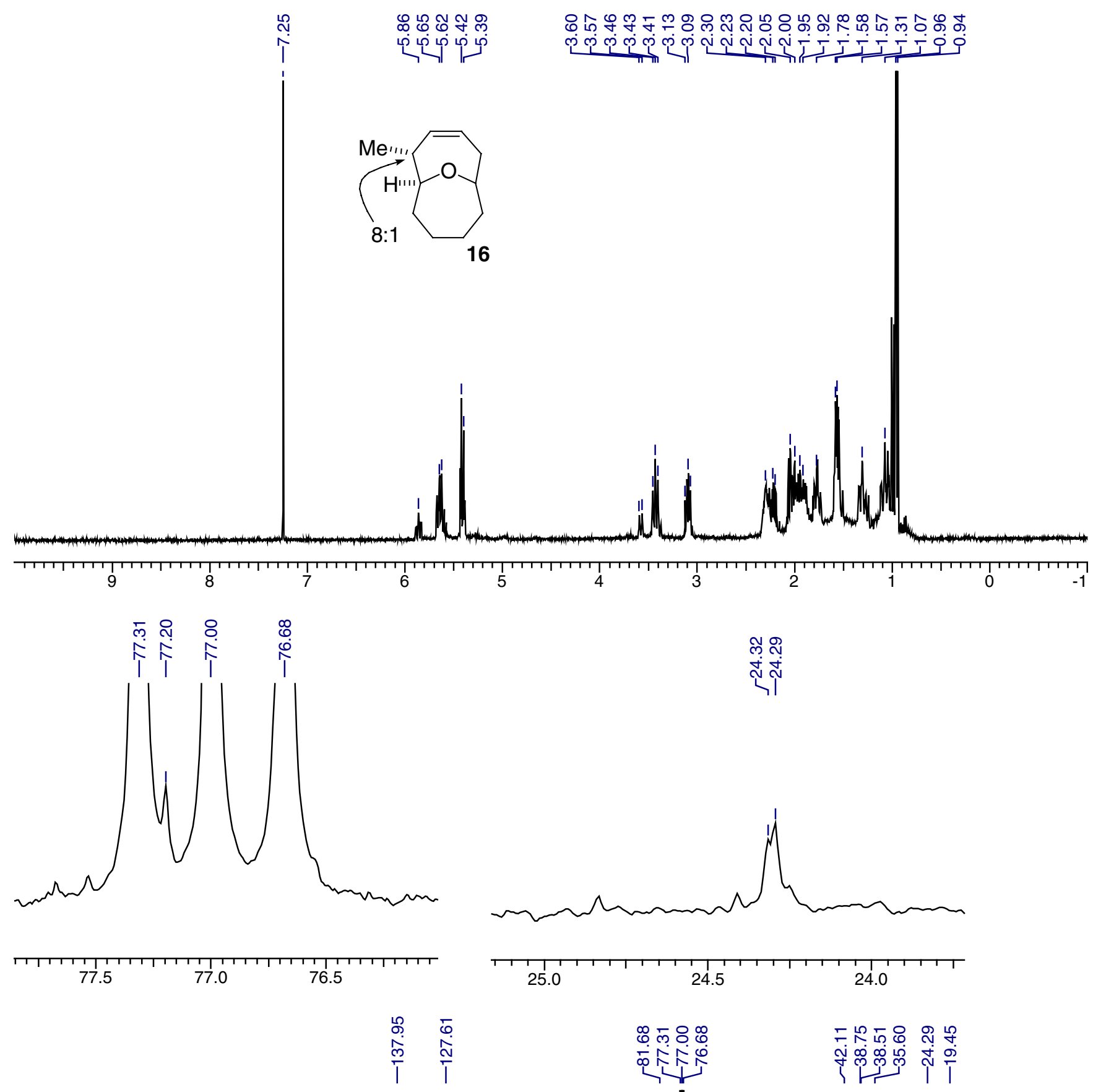

क्ष்

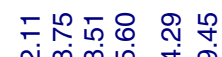

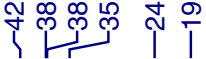
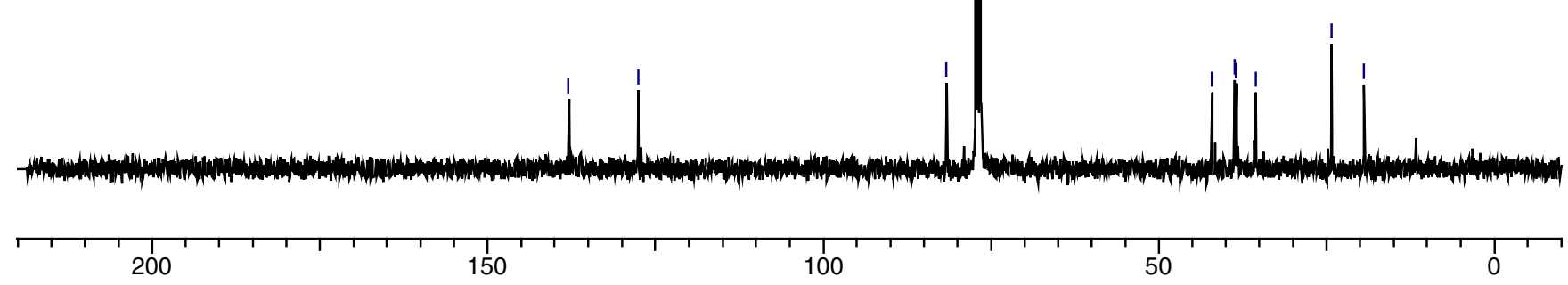

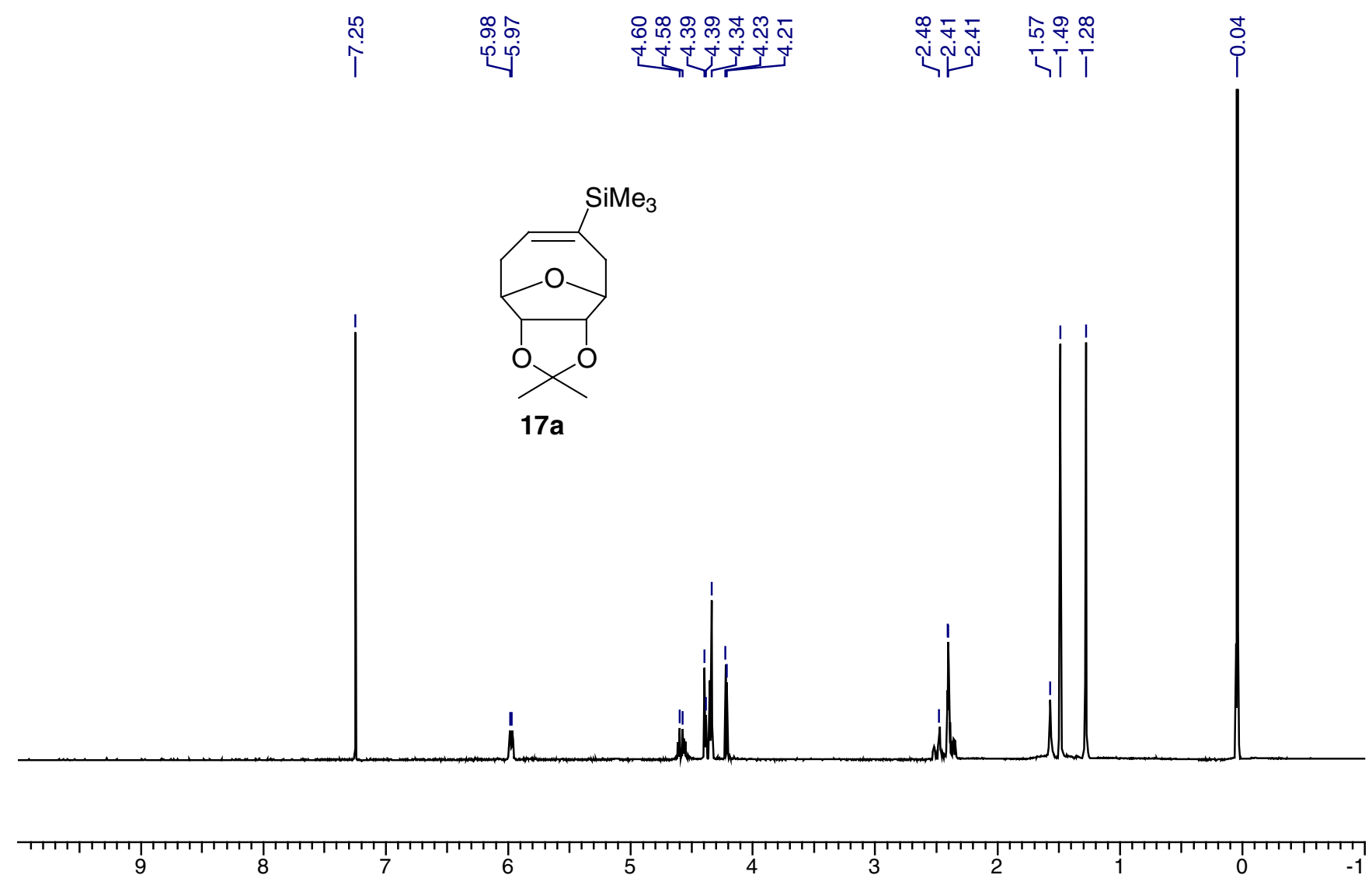

\begin{tabular}{|c|c|c|c|}
\hline 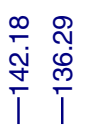 & 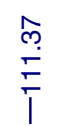 & 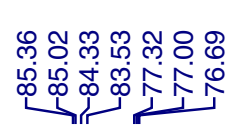 & 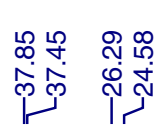 \\
\hline
\end{tabular}

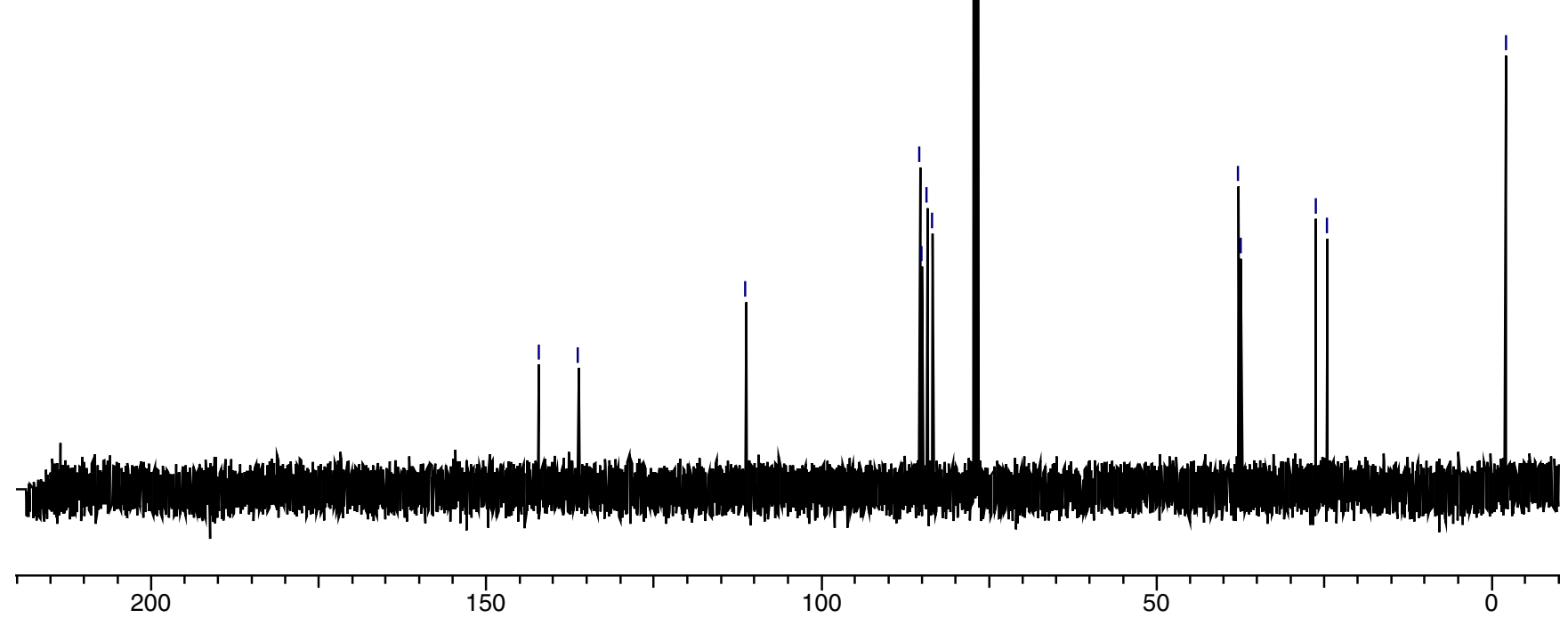




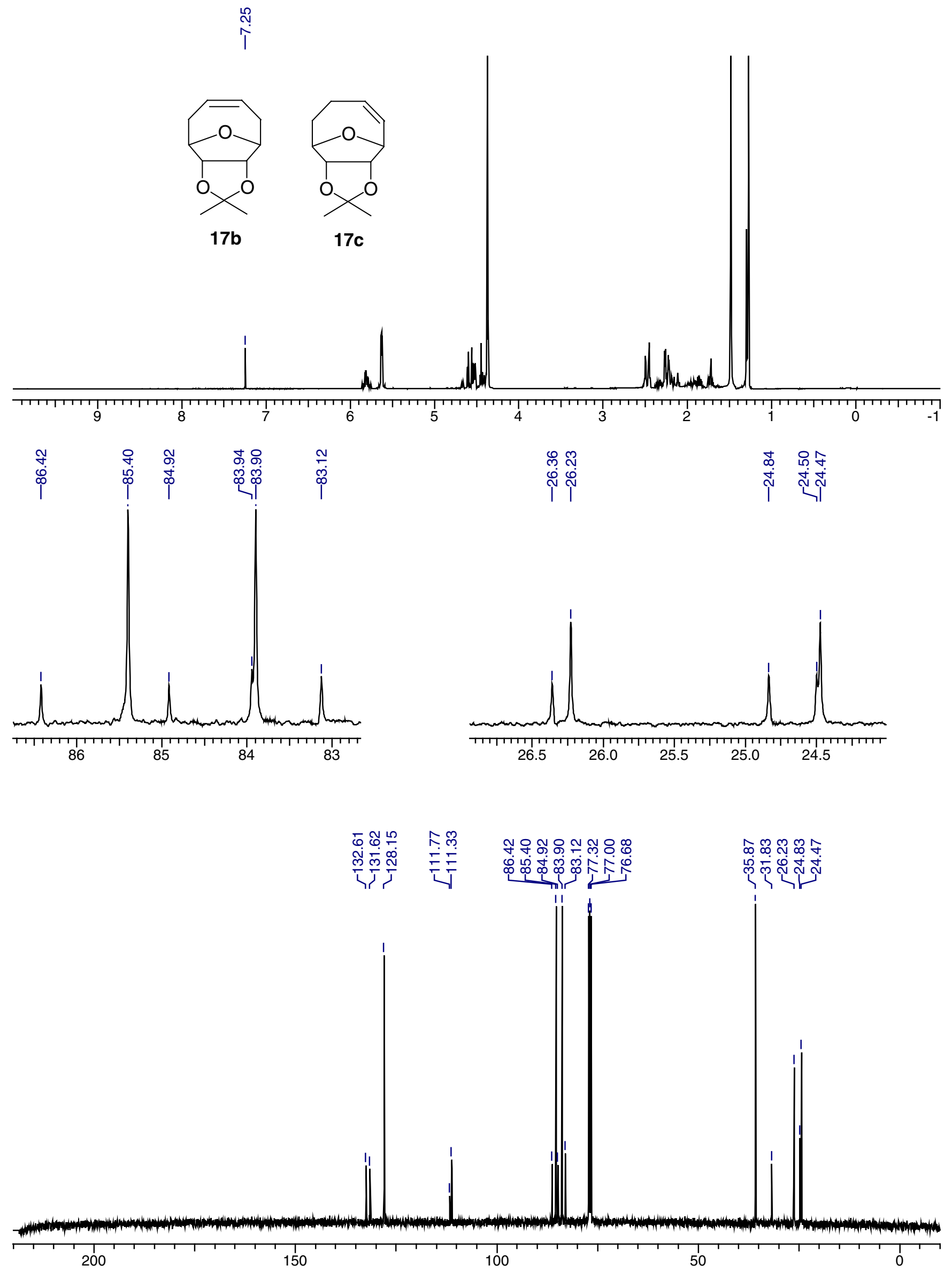




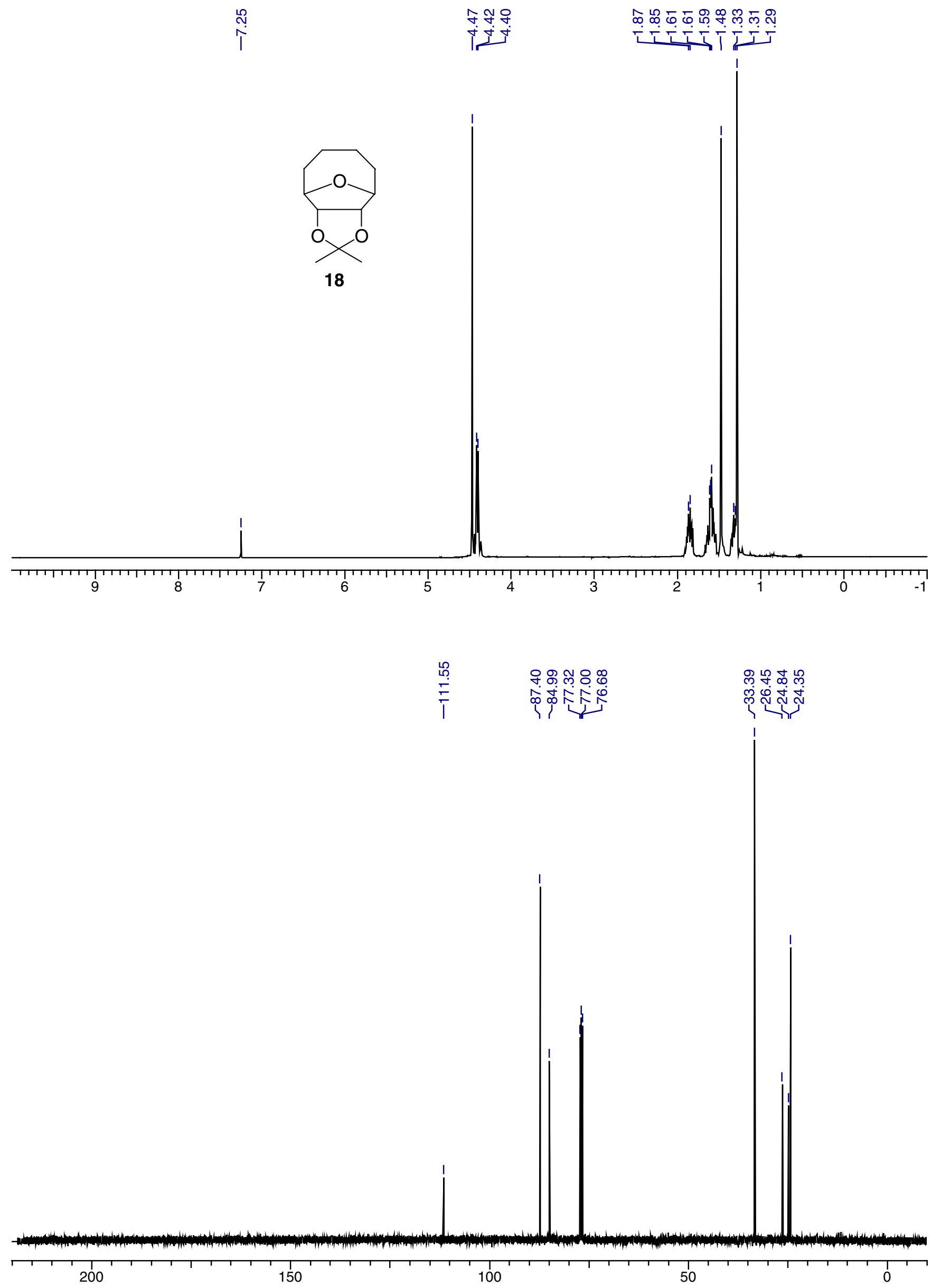




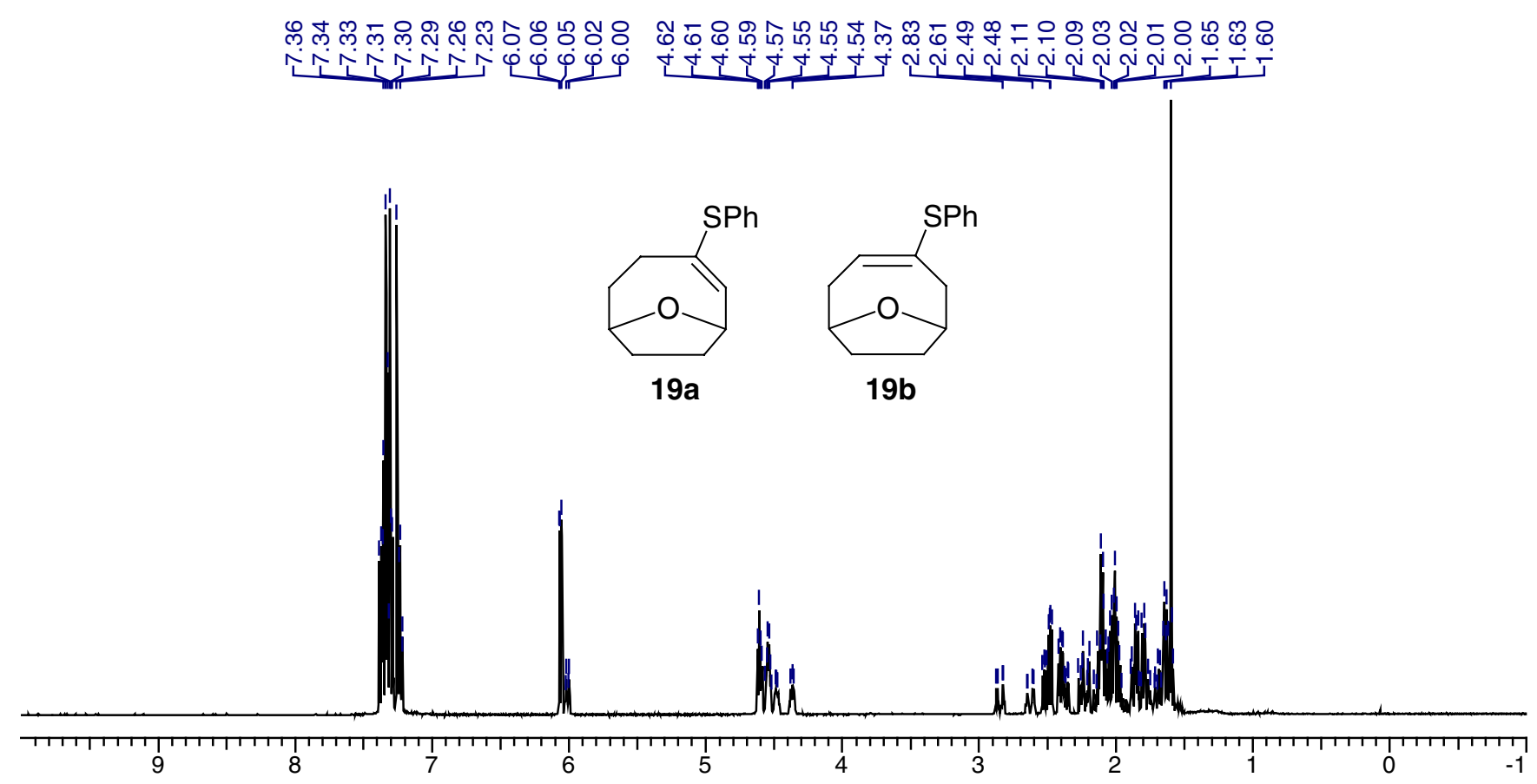

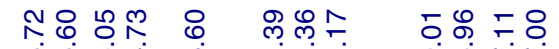

i

iٓ $\sum_{i}^{m}$ i

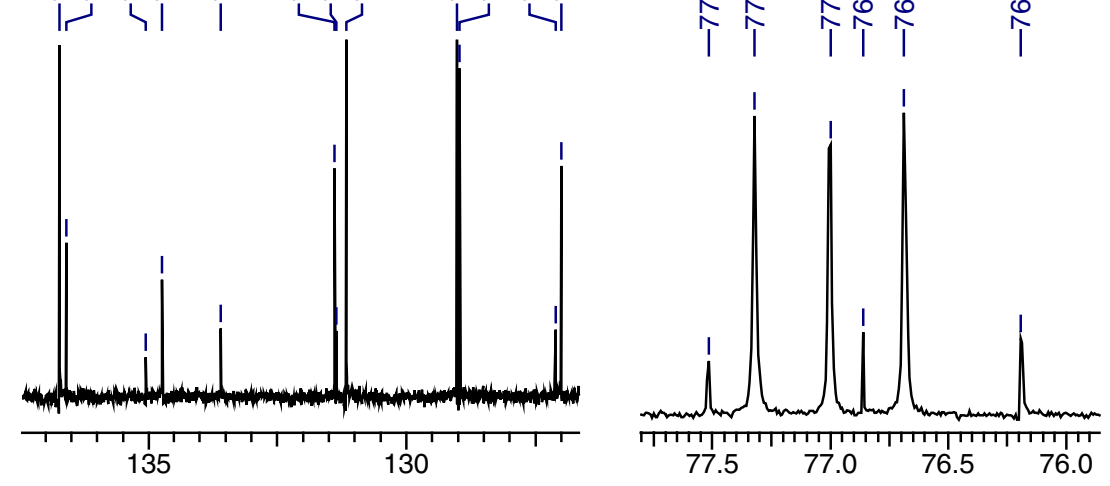

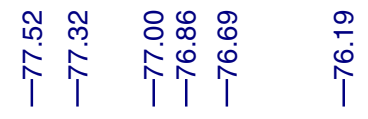

$\stackrel{\infty}{\bar{i}}$

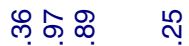

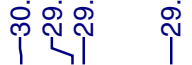

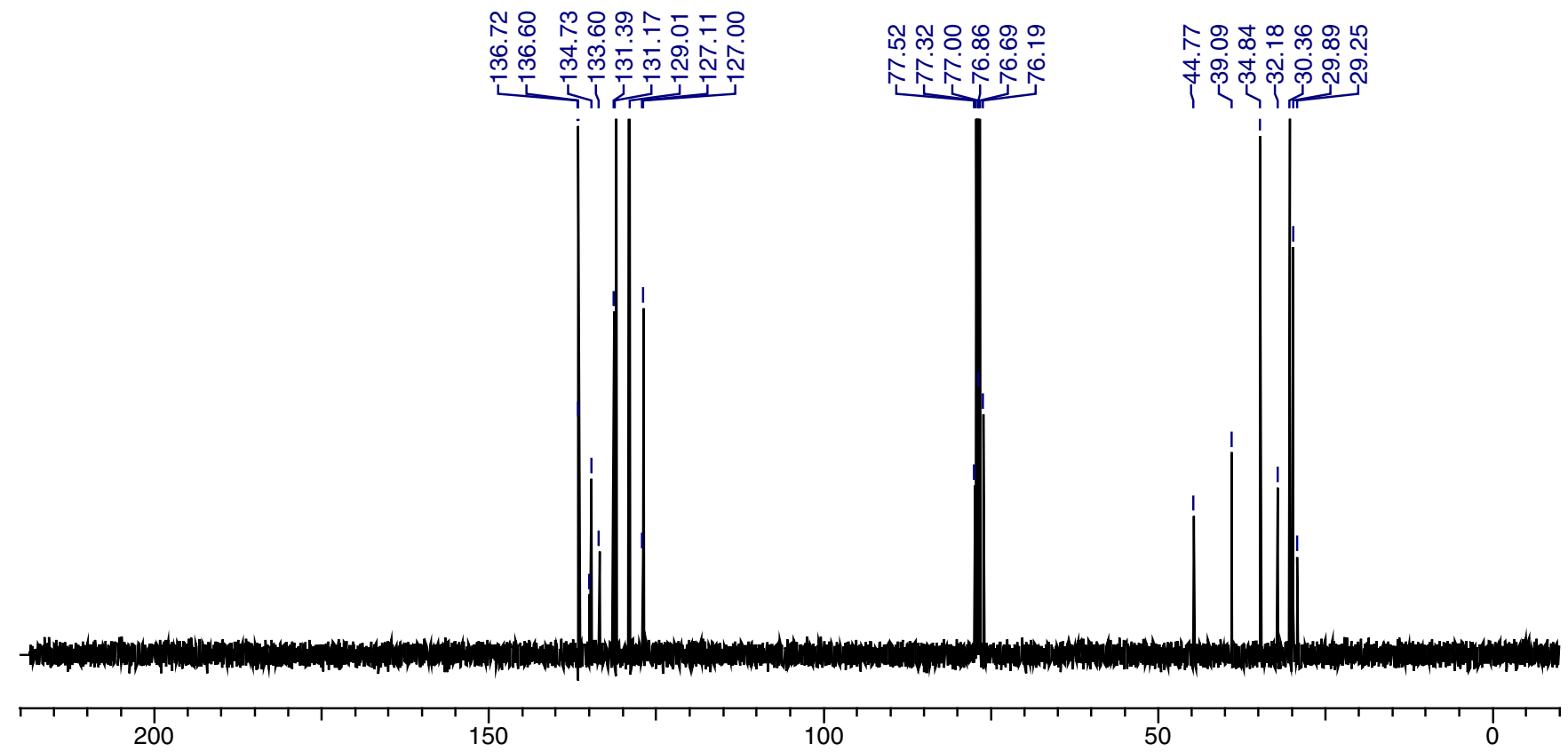



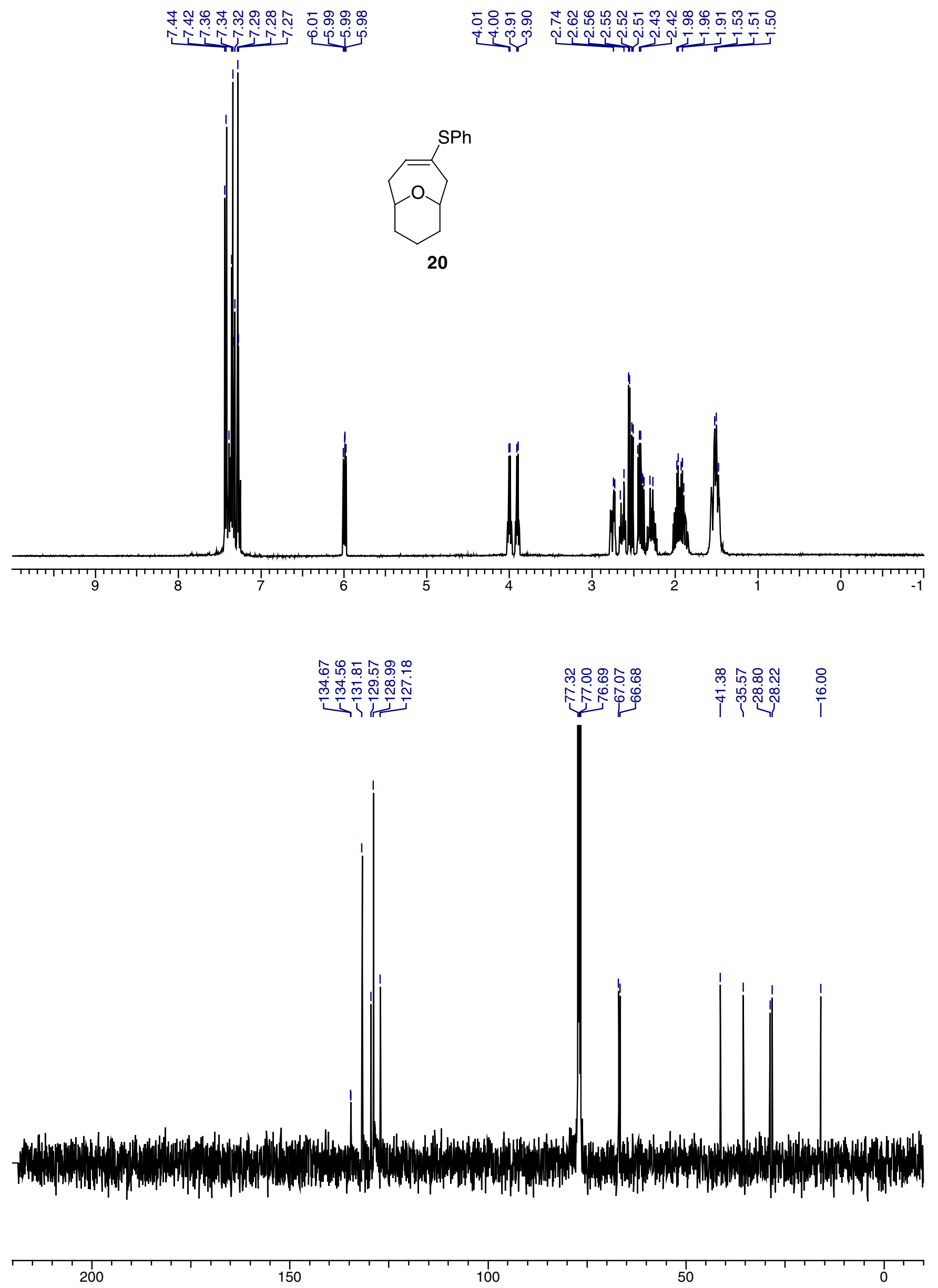

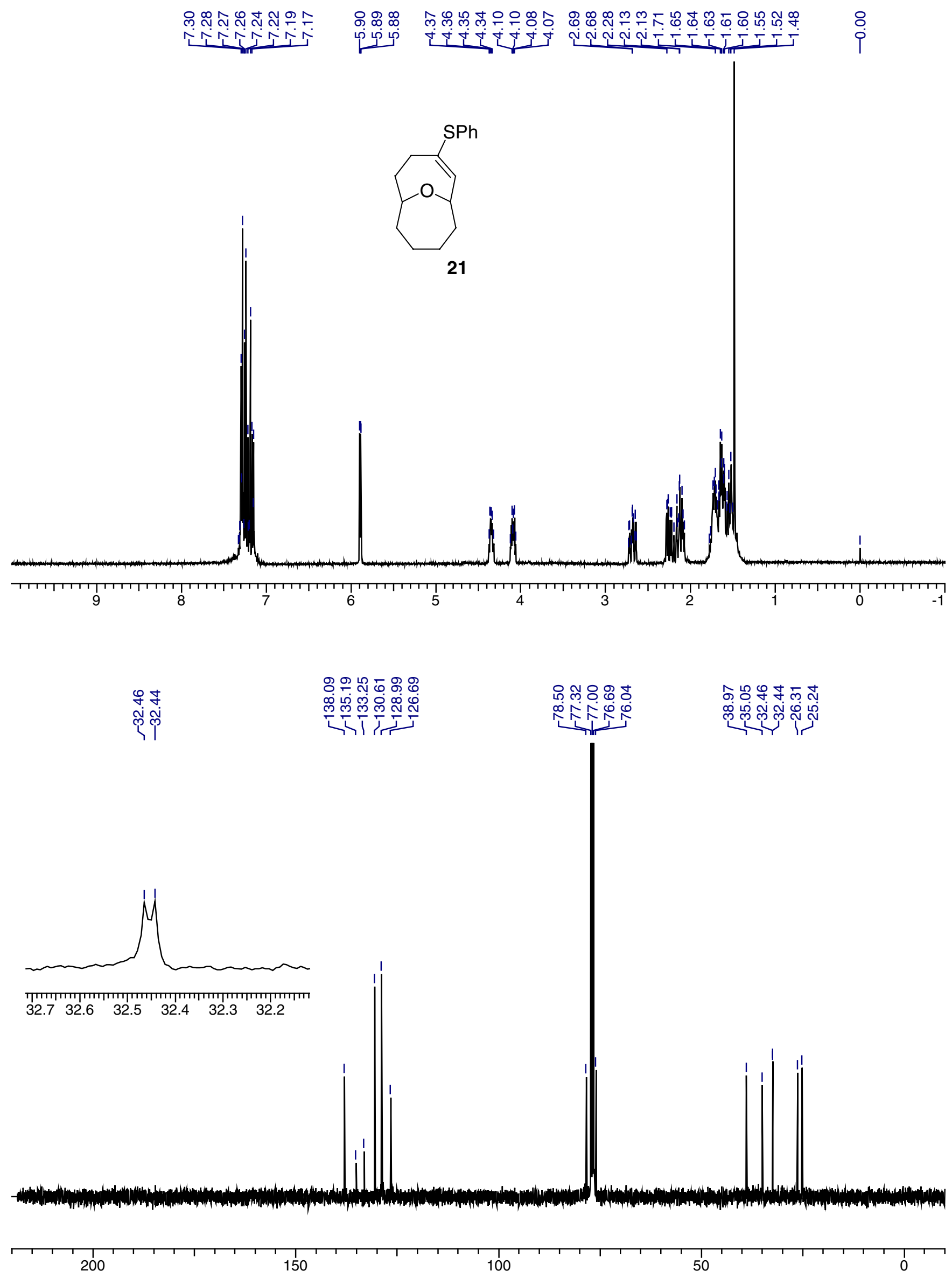

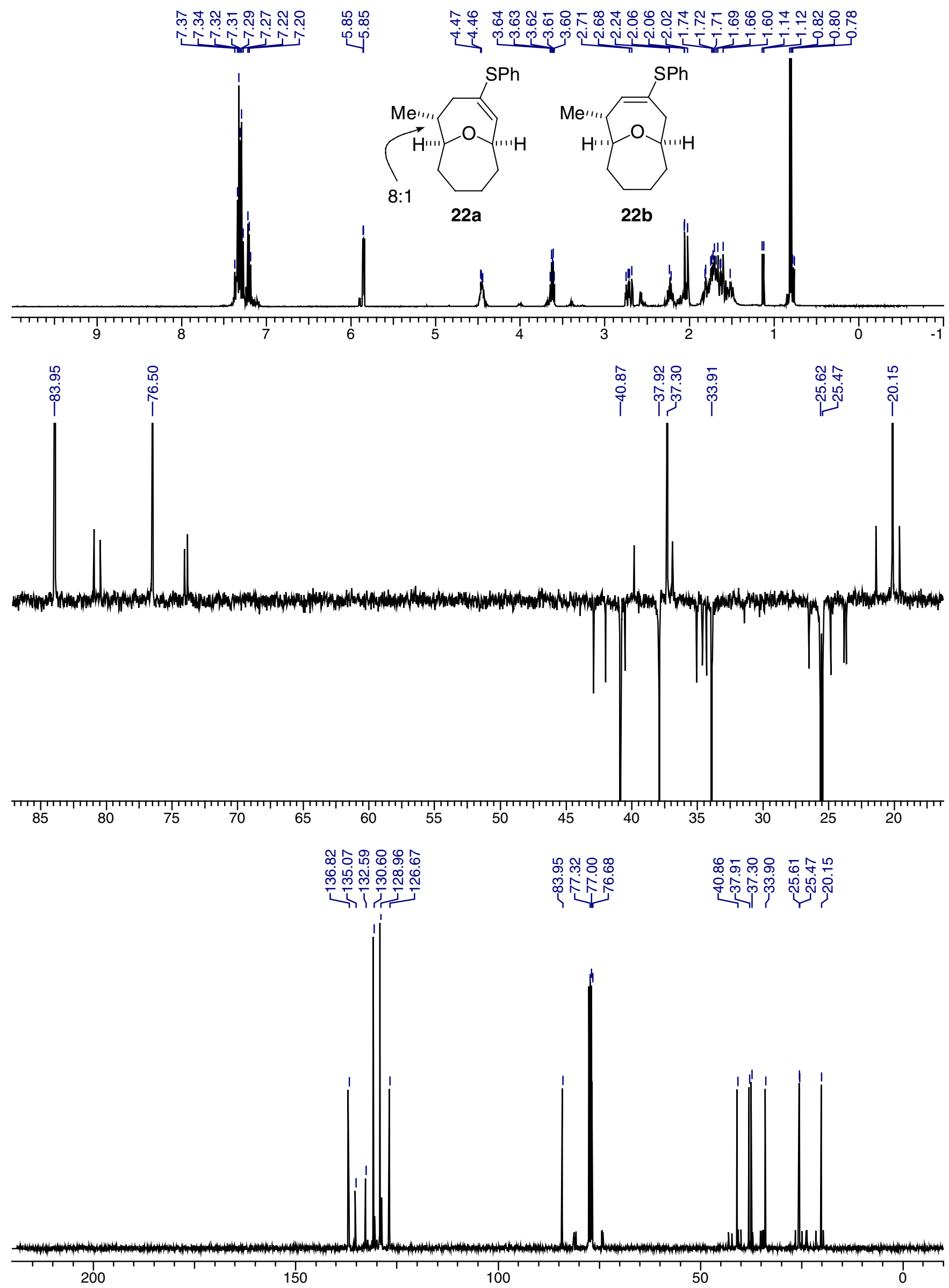


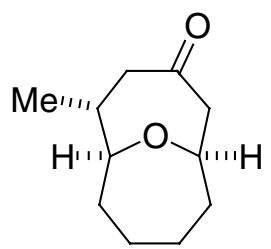

23
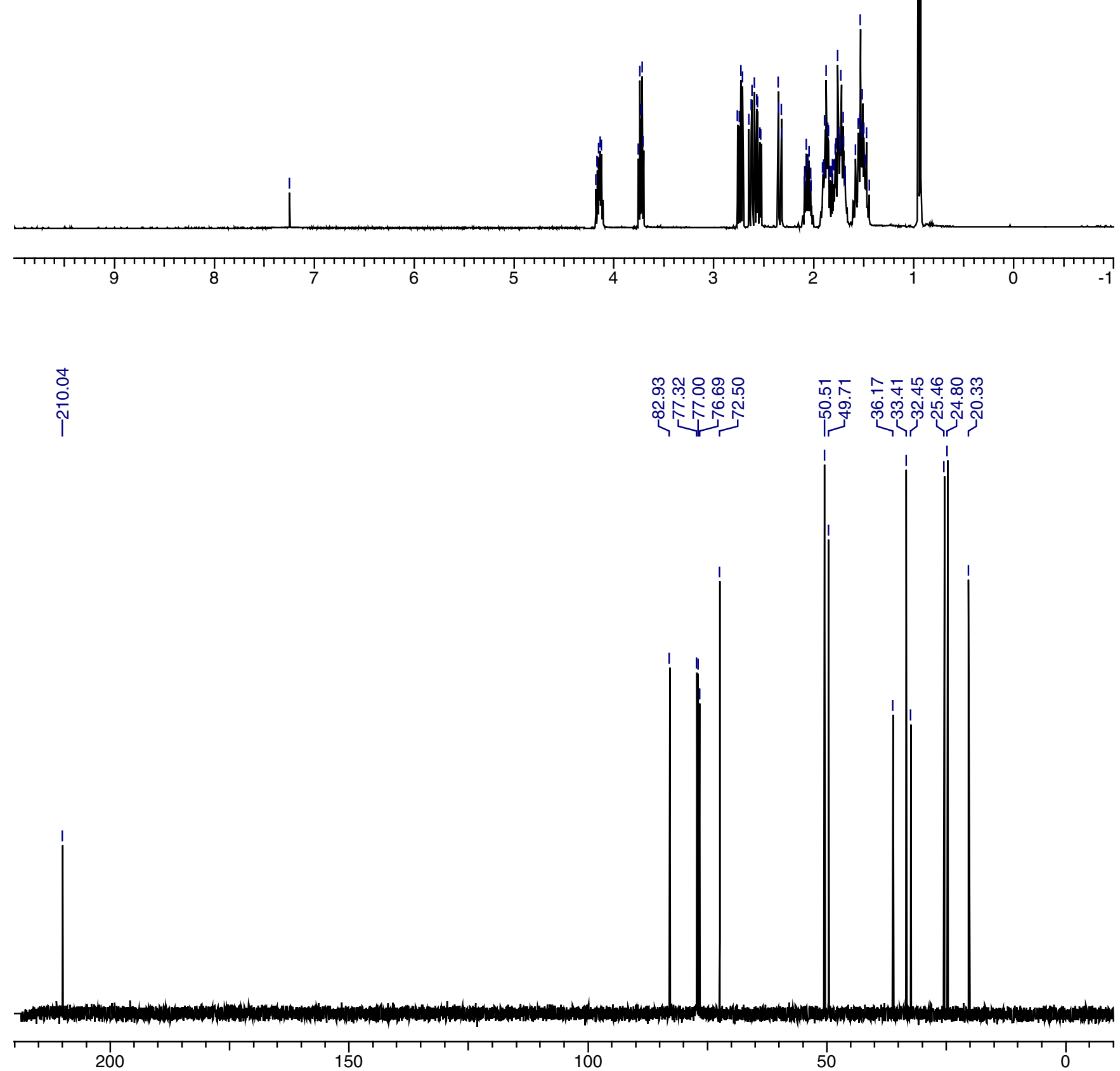

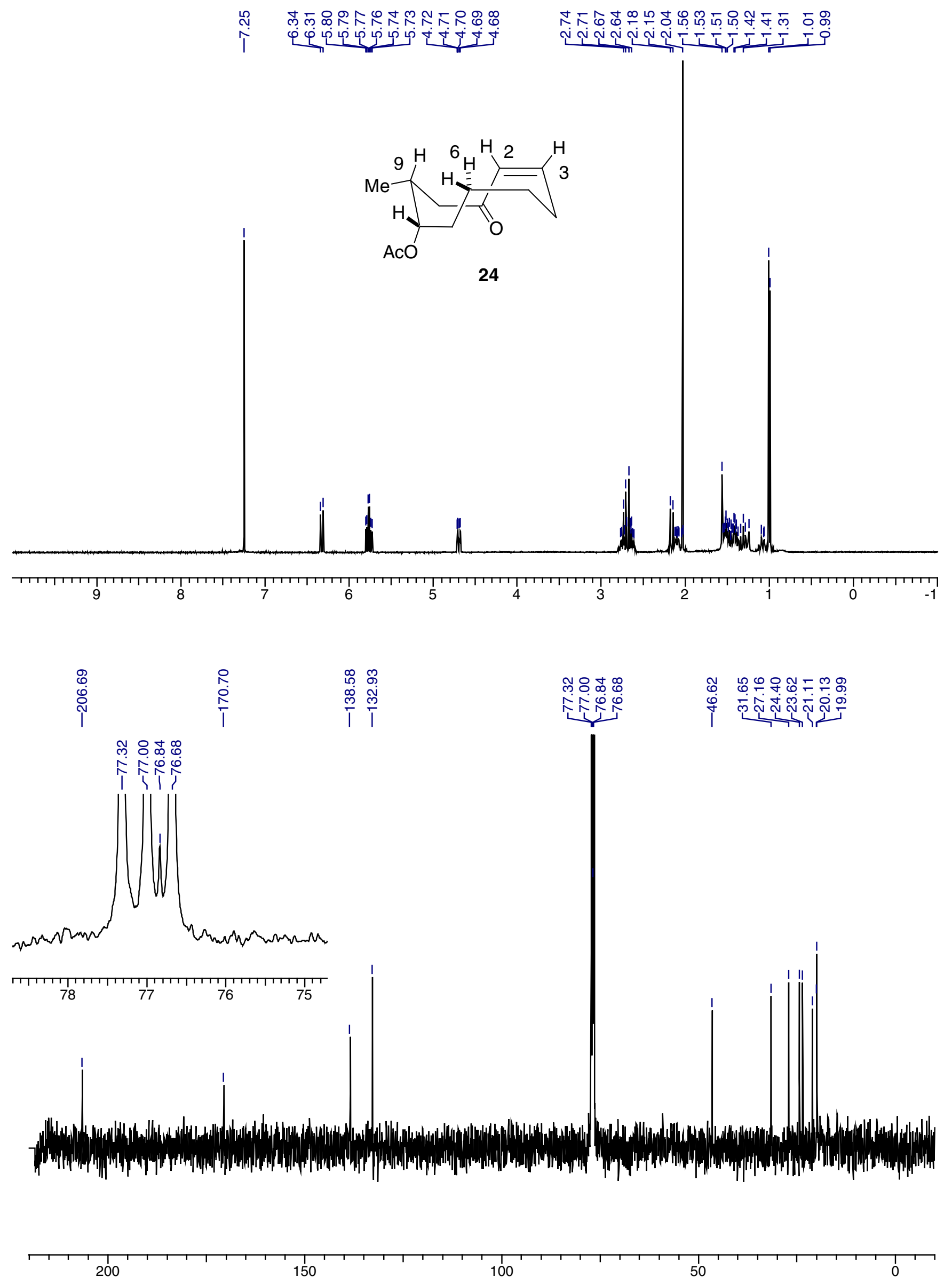


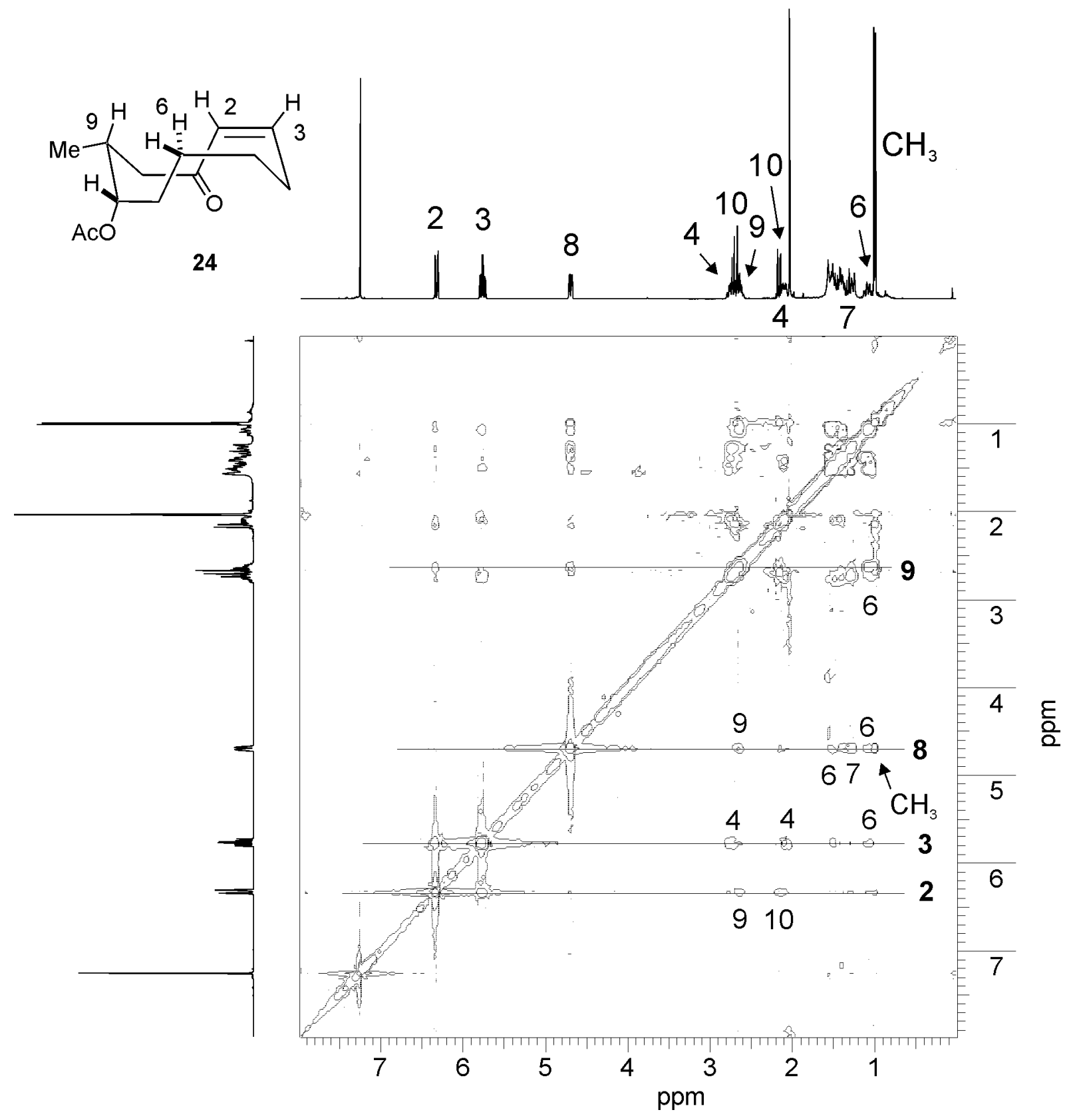

